Interlinguale Phraseologie

Theorie, Praxis und Perspektiven

Farø, Ken Joensen; Hallsteinsdottir, Erla

Published in:

Yearbook of Phraseology (2010)

Publication date:

2011

Document version

Også kaldet Forlagets PDF

Document license:

Ikke-specificeret

Citation for published version (APA):

Farø, K. J., \& Hallsteinsdottir, E. (2011). Interlinguale Phraseologie: Theorie, Praxis und Perspektiven. In Yearbook of Phraseology (2010) (Vol. 1, pp. 125-158). Mouton de Gruyter. 


\title{
Interlinguale Phraseologie: Theorie, Praxis und Perspektiven
}

\author{
ERLA HALLSTEINSDÓTTIR and KEN FAR
}

Abstract

In the following paper, several aspects of interlingual phraseology are considered. The term interlingual should be understood as an umbrella term (unlike, e.g. cross-linguistic or German kontrastiv), covering both theoretical and applied contexts within phraseology related to more than one language, but without necessarily being typologically focused. We also believe that there is a need for a unified linguistic theory of lexical units covering both phraseology (in the following we use the term phraseme for all phraseological units) and single words (Colson 2008, 2010; Dobrovol'skij and Piirainen 2005, 2009; Gries 2008). This paper is a contribution to such a theory of lexis.

Our definition of phrasemes, which serves to distinguish phrasemes from other lexical units in written language only, is based on the complexity and structure of the form of linguistic signs:

- Single words and - in languages like German, Icelandic and Danish - word formations: linguistic signs with a visually continuous form (cf. word definitions and the concept of a graphemic word in Fuhrhop 2008).

- Phrasemes: linguistic signs with a visually non-continuous form. Due to their non-continuous form, the sign character of phrasemes is not obvious in writing. Especially if a phraseme is not known by a speaker, this can create problems in applied contexts such as foreign language acquisition and translation.

The differentiation between graphemic words and phrasemes will result in either a need for a language specific categorization of phraseology (cf. Fleischer 1997: 249-250) or the inclusion of compound words in languages like French and English in the category phraseme. There are indeed, as Barz (2007: 27) points out, several functional, structural and semantic similarities between phrasemes and 
word formations (cf. also the discussion of Schwarzer Markt and Schwarzmarkt in Donalies 2009: 7-8).

Inspired by the diversity of recent phraseological research and the difficulties of describing our data with existing theoretical approaches based on the traditional linguistic dichotomy of langue-parole or performance-competence, we extend our linguistic approach to include a cognitive dimension (cf. Hallsteinsdóttir 2007: 161-162; Hallsteinsdóttir and Farø 2006). Thus we propose three approaches to phraseological research:

1. The study of phrasemes as a part of an abstract language system. We assume that the language system is the linguistic resource for language use.

2. The study of phrasemes in texts, where language is manifested as a result of language production that is based on a selection from the resources of the language system and, in part, determined by the linguistic knowledge of each speaker.

3. The study of phrasemes as a cognitive phenomenon that is a part of an individual speaker's linguistic knowledge and underlies idiosyncratic aspects of language processing based on the abstract language system.

This differentiation between forms of language manifestation is to some extent the base upon which the rest of the work is founded.

Functionalism is a central aspect of the way we look at phraseology in an interlingual perspective. This should be understood in two ways. First, we are interested in the functions of the phrasemes in the three manifestation forms of language and language use mentioned above. This includes discourse functions in general and the role-and communicative relevance-of iconography (images, pictures) in particular. Second, the idea of functionalism in this context involves a context specificity of many features which may seem relevant when analyzing phrasemes from a certain perspective within one linguistic discipline but which can be of no relevance in others.

We argue that all phrasemes have a complex semantic nature, which allows them to be used and understood both as compositional word sequences and as lexicalized units. This semiotic complexity provides an inherent potential for manipulations and it is a favored source for puns. Phrasemes are a part of the language system as lexicalized units. As well their constituents in most cases are also autonomous systemic units of the given language. The default textual realization of a phraseme is as a lexicalized unit since a compositional realization would automatically trigger the phraseological meaning, at least for known phrasemes. The cognitive linguistic processing of phrasemes can be either way: either based on the phraseological meaning for known phrasemes or on a com- 
positional processing of the meaning of the components - or even a combination of several strategies and meaning sources (cf. Hallsteinsdóttir 2001).

We assume that all phrasemes (like all lexical units in general) are conventional linguistic signs, and thus arbitrary, that is in the non-trivial, Saussurian reading of the word. The arbitrariness is a characteristic of the form-meaning relation in the linguistic system. Motivation, on the other hand, can be seen as a purely cognitive phenomenon based on the individual linguistic competence. Thus speakers are able to motivate phrasemes by (a) creating a relation between the form and the meaning of known phraseological units (retrospective motivation) and (b) interpreting the relation between the form and the meaning of assumed, but basically unknown phraseological units (prospective motivation) (cf. Farø 2006). The often purported (and even Saussure himself is not sufficiently consequent here) idea that arbitrariness and motivation are antonyms does not hold. Instead, we are dealing with two different perspectives on the same phenomenon.

Phraseological equivalence is a central issue in interlingual phraseology. As shown in figure 1, we propose that equivalence should be examined by including

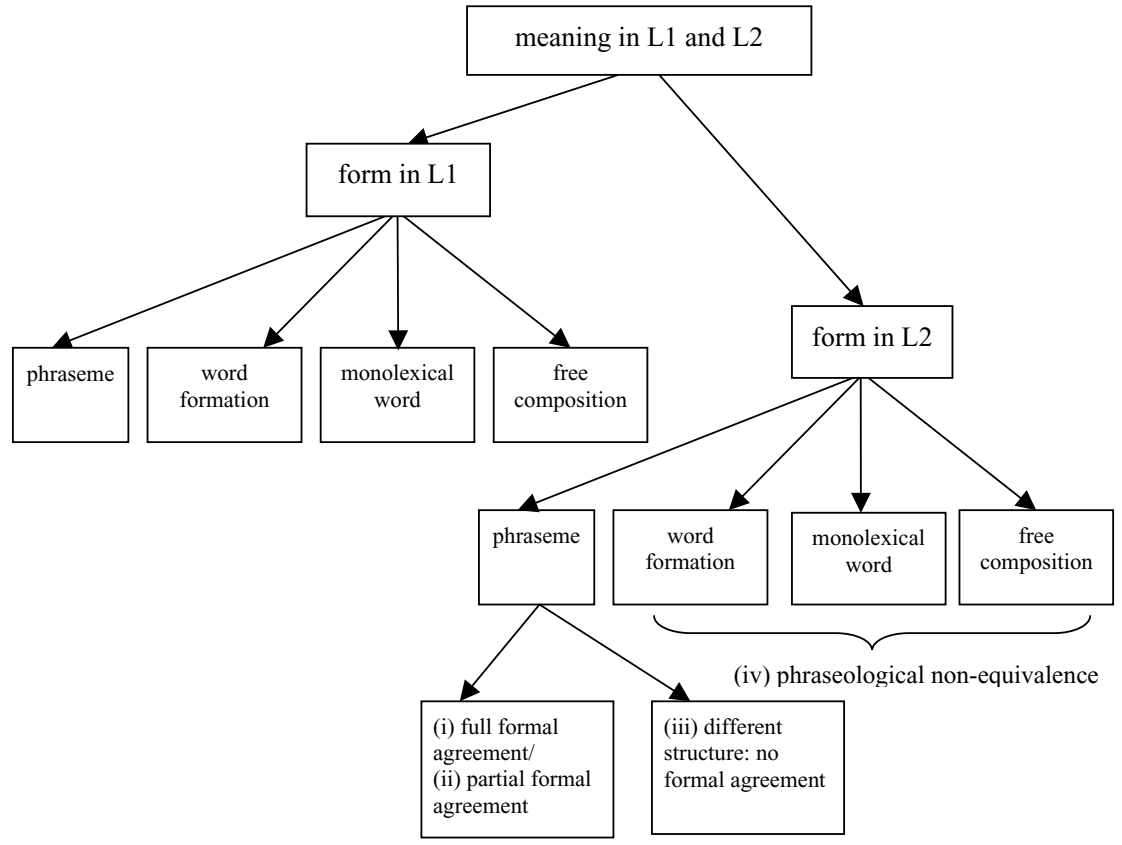

Figure 1. A holistic approach to equivalence of lexical items based on the types of phraseological equivalence in Korhonen and Wotjak (2001: 227) 
all lexical items instead of focusing only on equivalence within the phraseology of two or more languages.

We propose that a full understanding of equivalence within phraseology should not only include all lexical items but should also differentiate in the number and granularity of equivalence aspects (cf. Farø 2006) according to the respective research context. Each research context, and by that we mean linguistic discipline, demands its own specific theory and methodological approach of phraseological equivalence. Thus phraseology should be treated differently within various "applied" and one non-applied linguistic discipline and so four different approaches to phraseological equivalence in lexicology, lexicography, translation and language acquisition respectively are illustrated.

Keywords: phraseology; lexicology, lexicography; translation studies; language acquisition; interlingual linguistics.

\section{Einleitung}

Dieser Beitrag beschäftigt sich mit der linguistischen Beschreibung und Definition von Phraseologismen in der geschriebenen Sprache aus einer interlingualfunktionalistischen Perspektive. Das übergeordnete Ziel unserer Ausführungen ist es, der Tradition der kontrastiven Phraseologie folgend, einen wesentlichen Beitrag zu einer allgemeinen Theorie der Phraseologie zu liefern, denn schon seit den Anfängen der Phraseologieforschung hat die kontrastive Phraseologie neue, „auch intralinguale und allgemeinlinguistische Einsichten“ (Földes 2006: 11) und theoretische Erkenntnisse vermittelt.

Nach der Abgrenzung des Gegenstandsbereichs (Abschnitt 2) und einem kurzen Überblick über die interlinguale Phraseologie (Abschnitt 3) diskutieren wir grundlegende Fragen, die sowohl für die theoretische als auch die angewandte kontrastive Phraseologieforschung Relevanz haben. Unser Ausgangspunkt ist eine Differenzierung zwischen den unterschiedlichen Konstituierungsformen der Sprache als: (a) dem Resultat der Sprachverarbeitung in konkreten Texten, (b) der kognitiven Verarbeitung von Sprache und (c) dem abstrakten Sprachsystem. Wir skizzieren eine zeichentheoretische Grundlage, die auf unserer Dreiteilung basiert und die semantische Komplexität (Abschnitt 4) sowie die Rolle der Konventionalität, Arbitrarität und Motiviertheit (Abschnitt 5) in der Phraseologie mit einbezieht. Weiterhin legen wir dar, welche Aspekte die interlinguale phraseologische Äquivalenz ausmachen (Abschnitt 6) und inwieweit diese in den Disziplinen Lexikologie (Abschnitt 7.1), Translatologie (Abschnitt 7.2), 
Lexikographie (Abschnitt 7.3) und Sprachdidaktik (Abschnitt 7.4) theoretisch und praktisch implementiert werden können.

\section{Phraseologismen als verbale Zeichen}

Über die Eigenschaften der Phraseologie herrscht in der Forschung darin Einigkeit, dass Phraseologismen systemhafte sprachliche Zeichen sind, und dass die Merkmale der Phraseologizität auf allgemeingültigen Eigenschaften der Sprache beruhen (Burger et al. 1982: 315, 323). Als allgemein anerkannt gilt die Einteilung der Phraseologie in Phraseologie im engeren und weiteren Sinne. Alle Phraseologismen weisen die Merkmale Polylexikalität und Festigkeit auf, die sich in der durch die Lexikalisierung bedingten Reproduzierbarkeit des Phraseologismus als Wortgruppe manifestieren (vgl. Burger 2010: 15). Die Phraseologismen, die zusätzlich das Merkmal der Idiomatizität aufweisen, das die Abweichung der phraseologischen Bedeutung von der Komposition der Komponentenbedeutung eines Phraseologismus beschreibt, gehören zur Phraseologie im engeren Sinne (Burger 2010: 16; Burger et al. 1982: 1; vgl. eine kritische Auseinandersetzung mit weiteren Kriterien in Donalies 2009).

Es ist unumstritten, dass der Begriff Polylexikalität die lexikalische Komplexität eines Sprachzeichens bezeichnet. Diese Komplexität gilt für Phraseologismen, die aus Einheiten bestehen, die zum großen Teil selbst als selbständige Sprachzeichen fungieren (vgl. Häcki Buhofer 2006: x). Sie gilt aber auch für Wortbildungen, und um diese eindeutig von Phraseologismen abgrenzen zu können, ist es notwendig, die Konstituierung der Komplexität zu spezifizieren (vgl. Barz 2006, 2007; Fleischer 1997: 249-250). Wir schlagen vor, die Abgrenzung anhand der Beschaffenheit der Form vorzunehmen. Phraseologismen bestehen, im Gegensatz zu Wortbildungen, aus graphematischen Wörtern (Fuhrhop 2008). Die daraus zu ziehende Konklusion wäre, dass in der geschriebenen Sprache die primäre Aufteilung des Wortschatzes in Phraseologie und Wörter durch das distinktive Merkmal „Leerzeichen“ erfolgt:

1. Wörter: Wortbildungen und Simplizia, d.h. verbale Zeichen mit einer visuell wahrnehmbaren, einheitlichen Form.

2. Phraseologismen: verbale Zeichen, deren Ausdrucksseite durch Leerzeichen unterbrochen wird und die dadurch nicht unmittelbar als eine Einheit erkennbar sind.

Die Brauchbarkeit des Kriteriums Idiomatizität wurde inzwischen aufgrund seiner Subjektivität und der problematischen Operationalisierbarkeit mehrmals 
in Frage gestellt (vgl. die Übersicht in Donalies 2009: 20). Wir bevorzugen die Erfassung der semantischen Eigenschaften von Phraseologismen mit dem Begriff semiotische Komplexität (vgl. Abschnitt 5).

Wir gehen davon aus, dass Phaseologismen ein normales sprachliches Phänomen sind (vgl. Sinclairs ,idiom principle“, 1991, 2008), und dass sie als ein integrierter Bestandteil des Wortschatzes betrachtet werden sollten (vgl. Hallsteinsdóttir und Farø 2006). Unsere bisherige Forschung ${ }^{1}$ konzentriert sich v.a. auf die Phraseologie im engeren Sinne (Idiome), die somit als Grundlage unserer Ausführungen dient. Wir sind jedoch der Auffassung, dass viele der hier diskutierten Aspekte auch generell für die Phraseologie gültig sind, geben aber zu, dass wir noch nicht den empirischen Beweis dafür liefern können.

\section{Interlinguale Phraseologie}

Unter kontrastiver Phraseologie ${ }^{2}$ wird traditionell der Vergleich der Phraseologie in zwei oder mehr Sprachen verstanden. Die Ergebnisse aus kontrastiven Untersuchungen dienen sowohl zur Weiterentwicklung verschiedener Theorieansätze, z.B. der Sprachtheorie, der Erklärung der Verwendung von Phraseologismen, der Entdeckung universeller Prinzipien und der Beschreibung kultureller Muster, als auch als Grundlage von Arbeiten in angewandten sprachwissenschaftlichen Disziplinen (vgl. Colson 2008, 2010; Gries 2008; Korhonen 2007: 574-575).

Es wird zwischen diachronischen und synchronischen Dimensionen des Vergleichs unterschieden (vgl. im Folgenden Fleischer 1997: 241; Földes 2006: 11; Korhonen 2007: 574). Die historisch orientierten Arbeiten fokussieren meist auf die „Ermittlung der Herkunft, Verbreitung und Entlehnungsrichtungen phraseologischer Ausdrücke“ (Korhonen 2007: 574). In der synchron ausgelegten Forschung dagegen wurden bisher bevorzugt struktur- und komponentenbezogene Untersuchungen mit ,beschreibend-inventarisierender' Funktion durchgeführt, in denen bestimmte syntaktische und lexikalische Strukturtypen oder Sachgruppen der Komponenten, z.B. Tier-, Farb-, Körperteilbezeichnungen (Korhonen und Wotjak 2001) oder ganze phraseologische Systeme verglichen werden. Zur kontrastiven Phraseologie gehören ebenfalls Vergleiche, die sich auf die Kontrastierung anderer Phänomene wie Kultur, Symbolik und Figurativität konzentrieren, in die Phraseologismen als ein Teilbereich der Sprache einbezogen werden (vgl. Dobrovol'skij und Piirainen 2005; kurzer Überblick in Malá 2005).

Die methodische Vorgehensweise der bisherigen Untersuchungen kann grob in drei Verfahren eingeteilt werden, wobei der Komplexitätsgrad sehr unterschiedlich sein kann: 
1) Der auf der Intuition des Forschers basierende Vergleich von isolierten Phraseologismen oder phraseologischen Komponenten. Dieser bezieht sich häufig zusätzlich auf Informationen in ein- oder zweisprachigen Wörterbüchern. Dazu gehören auch der metalexikographische Vergleich und die Evaluierung der Darstellung von Phraseologismen in vorhandenen Wörterbüchern.

2) Der text- und korpusbasierte Vergleich von Phraseologismen. Den Ausgangspunkt bilden einerseits Phraseologismen in Wörterbüchern, die durch Beispielanalysen auf ihre Darstellung und Äquivalentangaben im Wörterbuch überprüft werden. Andererseits werden Phraseologismen in Übersetzungen auf das angewendete Übersetzungsverfahren untersucht. Als Grundlage dienen sowohl der Vergleich von Korpusdaten aus einsprachigen Korpora als auch der Vergleich von Originaltexten und Übersetzungen. Dazu kommen Untersuchungen gesprochener Sprache, die aber gerade im interlingualen Kontext zur Seltenheit gehören.

3) Der Vergleich von empirisch gewonnenen Sprecherdaten, die sich meist auf die Bekanntheit und Existenz von Phraseologismen, ihre Verwendung oder Verstehensprozesse in der Sprachverarbeitung beziehen. Diese Untersuchungen, die sich der Werkzeuge verschiedener Disziplinen wie der Statistik, der kognitiven Linguistik oder der Sprachlehr- und Sprachlernforschung bedienen, können sowohl einsprachige als auch mehrsprachige Probanden (Földes 2007) einbeziehen.

Recht früh hat man in der interlingualen Phraseologie zwischen einer Systemebene und einer Textebene unterschieden (vgl. z.B. Földes 1997). Diese Unterscheidung bezieht sich auf eine traditionelle sprachwissenschaftliche Dichotomie, die auf de Saussures Zweiteilung von langue und parole bzw. Chomskys Kompetenz und Performanz basiert (vgl. Korhonen 2007: 575). Korhonen und Wotjak (2001: 226) fordern jedoch eine stärkere Einbeziehung „neuere[r] Erkenntnisse der kognitiven Linguistik (z.B. zu den konzeptuellen Bereichen, die durch Phraseologismen abgedeckt bzw. nicht abgedeckt werden)“. Diese Forschungslücke ist auch schon z.T. geschlossen worden, denn zur kontrastiven kognitiven Linguistik gehören inzwischen empirische Untersuchungen zur Metaphorik und Figurativität, Kultursemiotik bzw. Kulturgebundenheit (vgl. Dobrovol'skij und Piirainen 2005; Malá 2005; Mellado Blanco 2010; Sabban 2007), zur Existenz, Bekanntheit und Verbreitung von Phraseologismen in verschiedenen Sprachen (Juska-Bacher 2009; Piirainen 2008³) und zur Phraseologie in der fremdsprachlichen Sprachverarbeitung (Hallsteinsdóttir 2001; Reder 2006, 2008).

Solchen Untersuchungen liegt meist eine Neuentwicklung von Methoden zugrunde und sie ergeben häufig ganz neue Datenmengen und Datentypen, 
die sich von bisherigen phraseologischen Daten deutlich unterscheiden. Die Unterschiede in der Beschaffenheit der Daten in ihren Untersuchungen nimmt Hallsteinsdóttir (2007: 161-162, vgl. auch Hallsteinsdóttir und Farø 2006) zum Anlass, die traditionelle sprachwissenschaftliche Dichotomie aufzubrechen und drei sprachliche Beschreibungsebenen anzunehmen.

Aufbauend auf dem Modell in Hallsteinsdóttir $(2007 ; 2010)$ und inspiriert durch Kilian (2001) erweitern wir die Zweiteilung der Sprache in System und Text durch eine sprecherbezogene kognitive Ebene der Sprachkompetenz:

1. Sprachsystem: die Sprache als ein abstraktes System von Regeln und Einheiten, in dem sowohl das synchrone als auch das diachrone Potenzial einer Sprache erfasst werden können. Die Beschreibung des Sprachsystems umfasst die inhärenten Realisierungsmöglichkeiten einer Sprache in Relation zur Konstituierung dieser Möglichkeiten in der Sprachverwendung und der Sprachkompetenz der Sprecher sowie eine Antwort auf die Frage danach, welche Faktoren die Realisierung bestimmter Möglichkeiten verursachen und andere blockieren. Das abstrakte Sprachsystem wurde schon in unterschiedlichen grammatischen Theorien in verschiedenen sprachtheoretischen Paradigmen beschrieben, in denen der Fokus jedoch meist auf einzelnen Wörtern und ihren Kombinationsmöglichkeiten liegt (vgl. Gries 2008). Zum Sprachsystem gehört ebenfalls eine linguistische Metaebene der Theorie und Methodik, die durch das gewählte sprachtheoretische Paradigma vorgegeben wird. Das Sprachsystem ist die Grundlage des Sprachgebrauchs. Es konstituiert sich im Gebrauch als die Basis für die Interaktion zwischen der Sprachverwendung und der Sprachkompetenz. Eine alleinige Realisierung des Sprachsystems als Text kommt jedoch nur in abstrahierten Beschreibungen z.B. in Wörterbüchern und Grammatiken vor.

2. Sprachverwendung: die Konstituierung des abstrakten Potenzials einer Sprache im Diskurs als ein Resultat der Sprachproduktion, d.h. die tatsächlich realisierten sprachlichen Konstruktionen. In der Sprachverwendung konstituiert sich Sprache im Gebrauch als ein Resultat der Interaktion von Sprachsystem und Sprachkompetenz in Form von konkreten Texten.

3. Kognitive Ebene der Sprachkompetenz: die Konstituierung des Potenzials einer Sprache als das - bewusste und unbewusste - Wissen des einzelnen Sprechers über Sprache sowie die in der Sprachverarbeitung eingesetzten mentalen Prozesse, d.h. die individuelle Sprachkompetenz (vgl. Hallsteinsdóttir 2001). Die Sprachkompetenz konstituiert sich im Gebrauch als eine Interaktion zwischen Text und Sprachsystem in der mentalen Aktivierung von Sprache in der Sprachproduktion und -rezeption. 
Hinter unserem Zugang verbirgt sich eine funktional-soziale Sprachauffassung, in der die Sprache als ein Gebrauchsphänomen ${ }^{4}$ betrachtet wird (vgl. Farø 2006). Die Art und Weise der Konstituierung der Sprache im Gebrauch und somit auch die Perspektive der linguistischen Beschreibung ordnen sich in die drei Dimensionen der oben beschriebenen Dreiteilung ein.

Die drei Beschreibungsebenen ermöglichen eine differenzierte Herangehensweise an die verschiedenen Konstituierungsformen von Phraseologismen, indem nicht nur die Systemebene und die Textebene, sondern auch die kognitive Ebene berücksichtigt wird. Die Vorteile dieser Aufteilung für die interlinguale Phraseologie sind, dass die Datengrundlage und ihre Bedingungen in Relation zu den Zielen der jeweiligen Arbeit genauer erfasst und beschrieben werden können.

\section{Semiotische Komplexität}

Die Lexikalisierung als verbale Zeichen mit einer konventionell festgelegten, einheitlichen Bedeutung führt bei allen Phraseologismen dazu, dass die phraseologische Bedeutung nicht der Addition der Bedeutungen der einzelnen Komponenten entsprechen kann, denn durch die Lexikalisierung wird die Komponentenbedeutung prinzipiell sekundarisiert und die Zeichenbedeutung des Phraseologismus tritt an ihre Stelle. Dadurch entsteht eine Komplexität auf der Inhaltsebene, die darin besteht, dass alle Phraseologismen ${ }^{5}$ - und nicht nur Idiome - potenziell mehrere Lesarten haben, d.h. mindestens eine kompositionelle und eine phraseologische. Es ist davon auszugehen, dass Sprecher jeder einzelnen Komponente eines Phraseologismus auch eigenes Bedeutungspotential zuweisen. Durch diese Bedeutungen haben sie die Möglichkeit, kompositionelle Bedeutungen zu konstruieren. Für Sprecher ist nicht die Zeichenhaftigkeit eines Phraseologismus, sondern die der einzelnen Komponenten visuell wahrnehmbar. Es ist daher anzunehmen, dass sowohl freie als auch phraseologische Bedeutungen in der Sprachverarbeitung potenziell verfügbar sind (vgl. ausführlich in Hallsteinsdóttir 2001: 45-52). Demnach sind auch alle Phraseologismen potenziell mehrdeutig, denn sie können potenziell kompositionell - wörtlich - verwendet und v.a. verarbeitet werden. Ersteres sieht man häufig in Sprachspielen und Letzteres dürfte nicht zuletzt bei unbekannten Phraseologismen der Fall sein.

Die Sprachkompetenz gaukelt uns zwar vor, wir verstünden bestimmte Typen von Phraseologismen - in der Forschung z.B. als nicht-idiomatisch bzw. transparent bezeichnet - durch ihre Komponentenbedeutungen. Ein Nichtmuttersprachler, der den Phraseologismus nicht kennt, wird die phraseologische Bedeutung häufig nicht richtig konstruieren können. ${ }^{6}$ So verbalisiert man die 
Bedeutung, die Zähne mit einer Zahnbürste reinigen' im Deutschen ${ }^{7}$ mit die Zähne putzen. Wer die phraseologische Bedeutung von die Zähne putzen jedoch nicht kennt, wird ohne Kontext durch die Bedeutungen von Zahn und putzen $\left(\right.$ vgl. DWDS ${ }^{8}$ ) nicht unbedingt unterscheiden können, ob und welche Art von Zahnpflege - die Benutzung von Zahnbürste oder Zahnseide, Zahnsteinentfernung, Beseitigung von Verfärbungen oder etwa professionelle Zahnreinigung gemeint ist:

1. ,etw. reibend, wischend blank machen`

2. ,etw. reinigen, säubern`

3. , sich, jmdn. schmücken, festlich kleiden`

Eine Monosemierung und somit die Festlegung der phraseologischen Lesart wird auf der Systemebene mit der Lexikalisierung und im mentalen Lexikon durch den Spracherwerb vollzogen. Die anderen möglichen Bedeutungen der Komponenten bleiben jedoch als Potenzial für kompositionelle Lesarten erhalten, wie im folgenden Beispiel:

- Im ersten Spiel konnten wir unseren Sieg verbuchen. Es ist ein guter Anfang, das [sic!] recht gut verlief. Hasan und Robert konnten je einen Tor schiessen [sic!]. Toll! ${ }^{9}$

Für welche Lesart von Tor - z.B. ,einfältiger, törichter Mensch` oder mit der Annahme eines grammatischen Fehlers: ,zum Hindurchgehen, Hindurchfahren bestimmte große Öffnung' bzw. ,mit einem Netz umspanntes, vorn offenes Gehäuse, in das der Ball von der Gegenpartei gebracht werden muß $\beta^{`}\left(\right.$ DWDS $\left.^{10}\right)-$ sich der Sprecher letztendlich entscheidet, hängt vom Kontext, Weltwissen und seiner Sprachkompetenz ab.

Die unterschiedlichen Lesartenmöglichkeiten lassen sich auf die drei Konstituierungsformen von Sprache beziehen (vgl. oben):

1. Sprachsystem: Das sprachsystematische Potenzial der einzelnen Komponenten, phraseologische Lesarten sowie evtl. logisch mögliche wörtliche Lesarten.

2. Sprachverwendung: Die tatsächlich im Text realisierten Lesarten.

3. Kognitive Ebene der Sprachkompetenz: Die in der Sprachverarbeitung konstruierten Lesarten.

Diese Auffassung von Lesarten ist deutlich weiter gefasst und unterscheidet sich somit grundlegend von bisherigen Ansätzen, die die kompositionelle 
Lesartenfähigkeit von logisch möglichen Interpretationen einer wörtlichen Lesart auf der Systemebene abhängig machen, d.h. wie z.B. Burger (2010: 62) Lesarten als „die möglichen semantischen Realisation einer bestimmten Wortverbindung" betrachten (vgl. auch Soehn 2006; Soehn und Römer 2007).

Unsere Untersuchungen haben gezeigt, dass Phraseologismen mit Ausnahme von Sprachspielen tatsächlich nur selten in einer kompositionellen wörtlichen Lesart vorkommen, d.h. die Konstituierung einer kompositionellen Lesart auf der Ebene der Sprachverwendung ist selten (vgl. Hallsteinsdóttir 2010), denn v.a. bei den frequenteren Phraseologismen ist anzunehmen, dass die phraseologische Bedeutung salient ist und somit eine rein wörtliche Lesart unmöglich macht. Aus diesem Grund sollte möglicherweise eher von einem Lesartenkontinuum ausgegangen werden, das von einer rein wörtlichen bis zu einer rein phraseologischen Lesart reicht: ${ }^{11}$

- Eskortiert von Fangschiffen verließ die „Rainbow Warrior II“ im Kielwasser eines Schleppers der Meerespräfektur die Bucht von Marseille in Richtung Spanien.

- Lindemann hatte sich 1956 in ein Faltboot gesetzt und im Kielwasser Bombards über den Atlantik begeben.

- Pünktlich zum Erscheinungstermin des sechsten Bandes kam die etwas angestaubte Korrespondenz jetzt an die Öffentlichkeit - im Kielwasser eines mächtigen PR-Kreuzers, der mit immer neuen Gags die globale Hysterie auf Touren hält.

- Die deutschen Technologiewerte sind am Donnerstag im Kielwasser positiver Vorgaben aus den USA gut behauptet in den Handel gestartet.

Die semiotische Komplexität bedingt eine prinzipielle Modifizierbarkeit von Phraseologismen und sie kommt häufig durch Relationen zu und das Zusammenspiel mit anderen Zeichensystemen zum Vorschein. Die Realisierung von Modifikationen korrespondiert möglicherweise mit den in der Forschung beschriebenen logischen Lesarten, d.h. je logischer die wörtliche Lesart ist, desto einfacher lässt sich ein Phraseologismus modifizieren. Hierzu sind jedoch weitere Untersuchungen erforderlich.

In Modifikationen spielt das in der Forschung als Bildhaftigkeit, Bildlichkeit oder Ikonographie (vgl. Farø 2006) genannte Phänomen eine große Rolle. Die Ikonographie ergibt sich z.B. durch die Bedeutungen der einzelnen Komponenten, kulturelle Symbole und Weltwissen der Sprecher. Die folgenden Beispiele zeigen einige Realisierungsmöglichkeiten der semiotischen Komplexität.

In der Überschrift Island taget ved hornene („Island an den Hörnern gepackt") ist die verbale Komponente tyr (,Stier') durch Island ersetzt worden und 


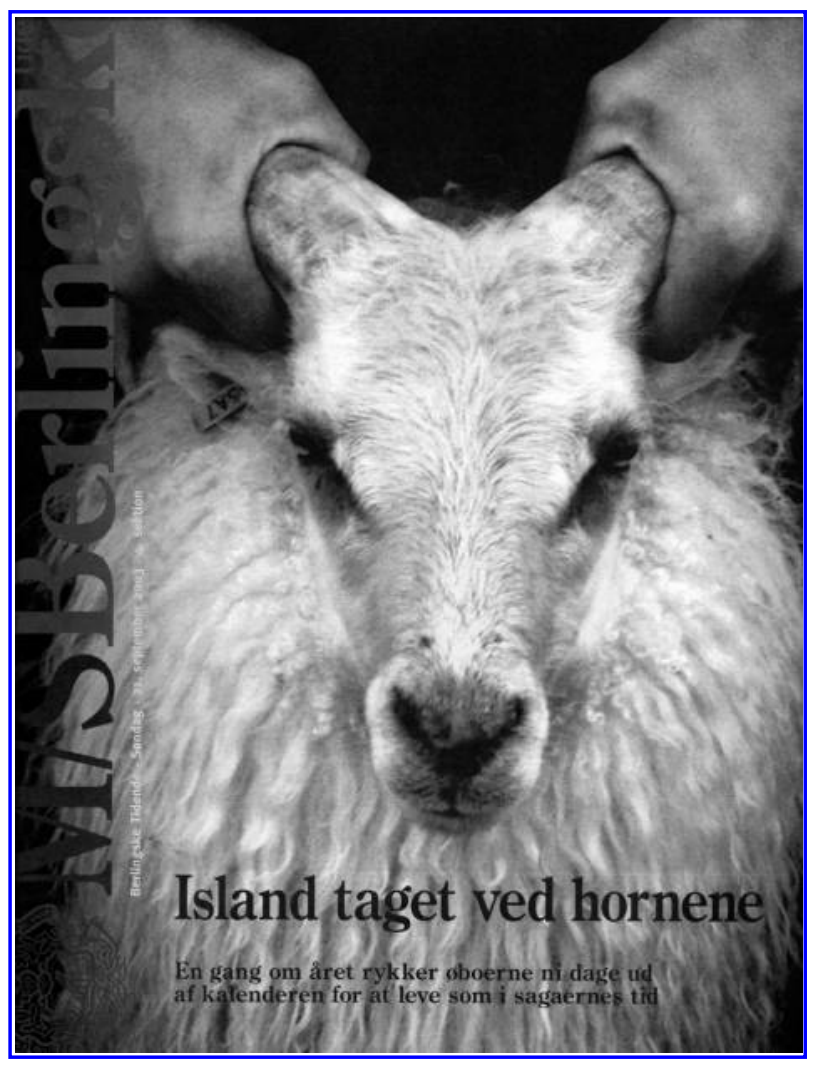

Abbildung 2. Berlingske Tidende, 21. September 2003

die Auswahl des visualisierten Tieres (junger Schafsbock) erfolgt in Relation zu dem im Text behandelten Thema: der im Herbst stattfindende Schafabtrieb in Island.

Im zweiten Beispiel (Abbildung 3) kann zunächst eine konkrete Visualisierung der Komponente Stier in der Überschrift vermutet werden. Ein Blick auf die untergeordnete Überschrift verrät jedoch, dass der Stier auch die Frankfurter Börse symbolisieren könnte, deren Wahrzeichen bekanntlich Skulpturen von einem Bullen (für steigende Kurse) und einem Bären (für fallende Kurse) sind. Die beiden englischsprachigen Beispiele zeigen verschiedene Stier-Typen. Abbildung 4 zeigt einen gefährlichen, angriffslustigen Stier, mit dem die Firma mit ihren mutigen Rettungsmaßnahmen den Kampf aufnehmen will. Bei der Skulptur handelt es sich beim genaueren Hinsehen um das Symbol der Börse 


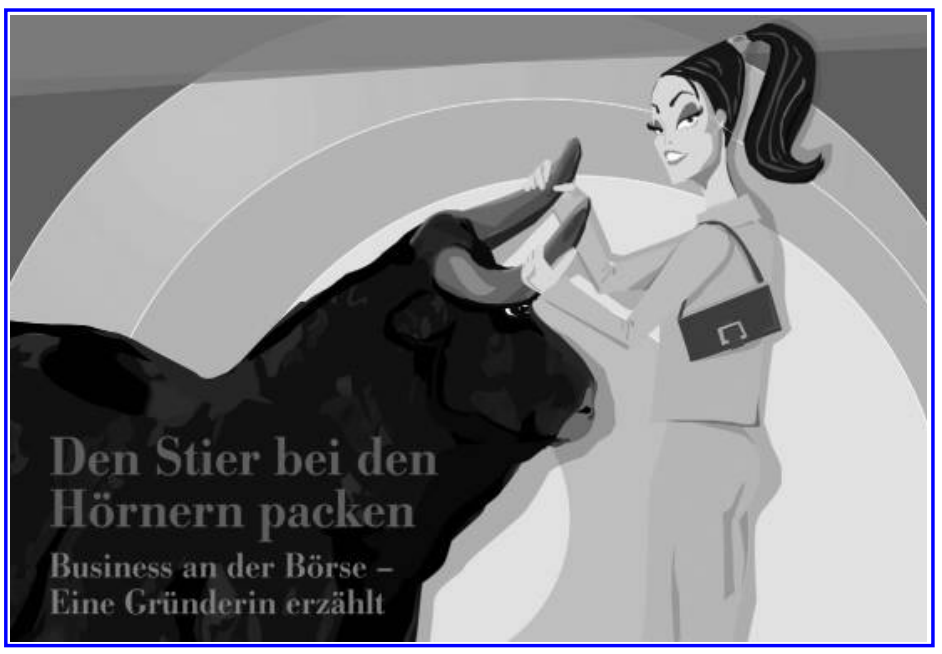

Abbildung 3. Gründerelle. Das Magazin für Gründerinnen, Ausgabe 2 www.wirtschaftsfoerderung-dortmund.de/tiny/oj/ (30. März 2010)

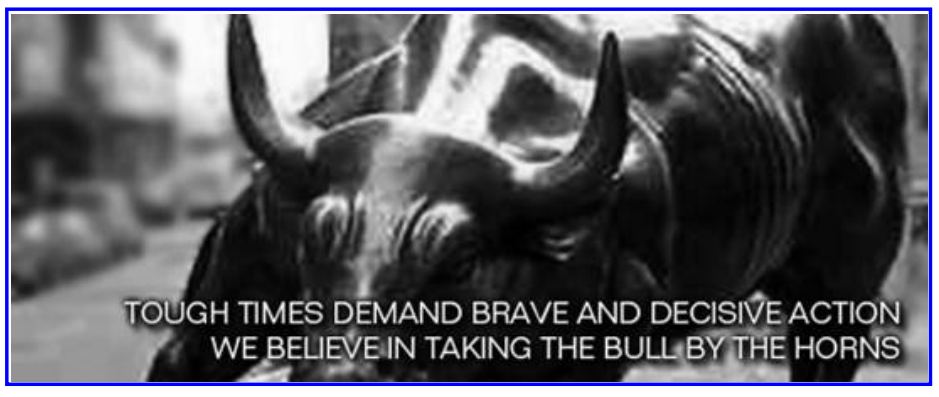

Abbildung 4. Werbung von BanxQuote ${ }^{\circledR} R_{x}$. Business Debt Restructuring Solutions www.banx.com/images/bullb\&w.jpg (30. März 2010)

in Wall Street, wodurch ein direkter Bezug zu den aktuell schweren Zeiten der Finanzwelt herstellbar wird.

Im zweiten Beispiel (Abbildung 5) sehen wir einen durch einen Nasenring domestizierten und unter Kontrolle gehaltenen Zuchtbullen. Die abgeschlossene Zähmung und die Macht über das Zuchttier werden durch das Festhalten der Hörner demonstriert: ${ }^{12}$

Das im Weltwissen der Sprecher verankerte Symbolikwissen, das im deutschsprachigen und dem ersten englischsprachigen Beispiel das Verstehen beeinflusst, kann für den dänischen Phraseologismus nicht eingesetzt werden, denn die 


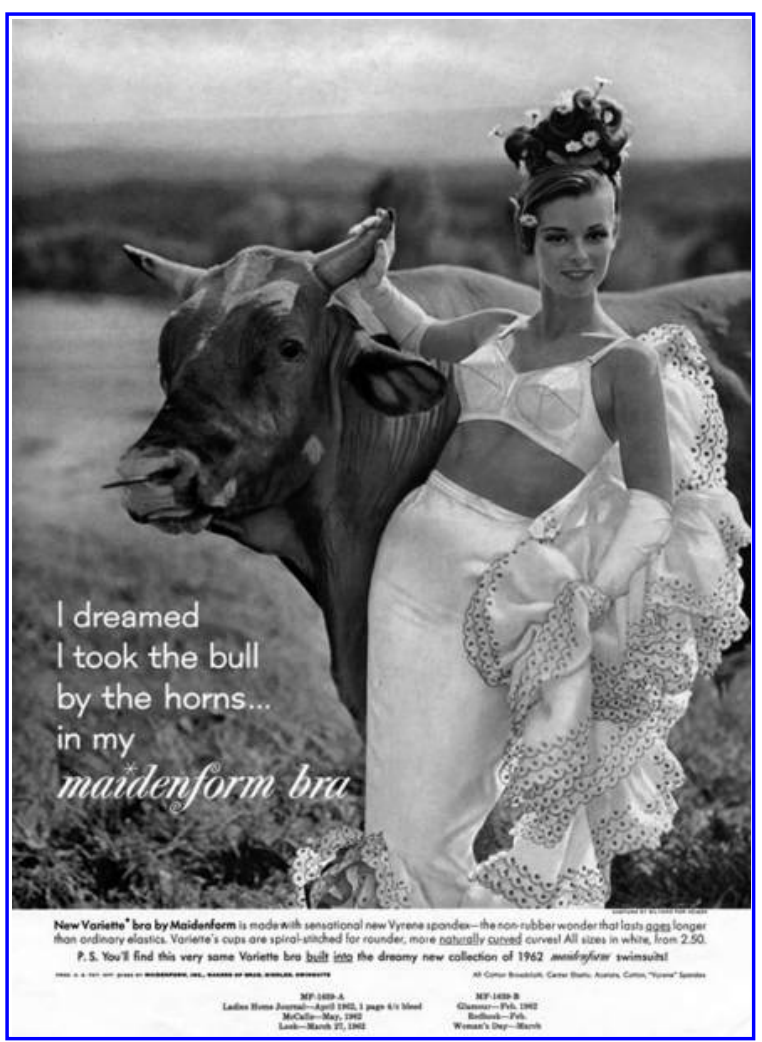

Abbildung 5. Werbung für Unterwäsche aus dem Jahr 1962

Komponente Stier/Bulle hat keinen vergleichbaren Symbolwert im Dänischen. Im Isländischen gibt es keinen entsprechenden Phraseologismus.

Die semiotische Komplexität und die sich daraus ergebende potenzielle Mehrdeutigkeit und prinzipielle Modifizierbarkeit von Phraseologismen ist, zusammen mit der Unauffälligkeit der Form, eine der Haupterklärungen für Probleme sowohl in der theoretischen Beschreibung als auch der angewandten kontrastiven Phraseologie (vgl. eine ausführliche Argumentation dazu in Farø 2006).

\section{Arbitrarität und Motiviertheit}

Als zeichentheoretischer Ausgangspunkt dient die bilaterale Saussure'sche Auffassung, dass die Zuordnung von Ausdruck und Bedeutung arbiträr ist und von den Sprechern einer Sprachgemeinschaft ausgehandelt wird. Das Prinzip der 
Arbitrarität sprachlicher Zeichen in dem Sinne, dass es keinen zwingenden $\mathrm{Zu}-$ sammenhang zwischen der Ausdrucksseite und der Bedeutung gibt, ist eine wichtige Grundlage aller kontrastiven Analysen (vgl. Farø 2006). Demnach ist weder die Bedeutung noch die Ausdrucksseite eines Zeichens durch das jeweils andere vorhersagbar. Auf die Phraseologie bezogen bedeutet dies, dass die Kombinierbarkeit der einzelnen Komponenten zu einem Phraseologismus nicht durch die phraseologische Bedeutung bestimmt wird und umgekehrt. Die Konsequenz ist, dass nicht von der Ausdrucksseite eines Phraseologismus auf die Ausdrucksseite eines Phraseologismus in einer anderen Sprache und ebenfalls nicht von der Ausdrucksseite auf die Äquivalenz von Phraseologismen und auch nicht auf die Äquivalenz einzelner Komponenten geschlossen werden kann (vgl. Farø 2006; Reder 2006). Der interlinguale Beweis der Arbitrarität sind die falschen Freunde, d.h. Phraseologismen, die ,auf der Ebene der lexikalischen Konstituenten und mentalen Bilder, d.h. auf der Ebene der inneren Form“" (Dobrovol'skij und Piirainen 2009; 147) Ähnlichkeiten aufweisen, jedoch unterschiedliche Bedeutungen haben.

Eine mit dem Arbitraritätsprinzip eng verbundene Konzeption ist die Motiviertheit bestimmter sprachlicher Zeichen. Die Motiviertheit besagt, dass bei bestimmten Zeichen doch eine Relation zwischen Form und Bedeutung besteht.

Es ist wichtig festzuhalten, dass sich die Motiviertheit und Arbitrarität nicht gegenseitig ausschließen, sondern zwei verschiedene Phänomene sind, die zwei verschiedene Perspektiven auf Sprache erfordern. Die Arbitrarität ist eine auf das Sprachsystem bezogene theoretische Voraussetzung. Die Motiviertheit bezieht sich dagegen auf die Sprachkompetenz der Sprecher. Die Motiviertheit teilen wir übergeordnet in drei verschiedene Phänomene auf (vgl. Farø 2006), die auf verschiedenen Strategien basieren können (vgl. Hallsteinsdóttir 2001).

Die Entstehungsmotiviertheit bezieht sich auf das Wissen um die Benennungsmotivation/-prinzipien. Sie ist diachron im Sprachsystem verankert und kann i.d.R. nur von Linguisten nachvollzogen werden. Dies gilt beispielsweise für den Ursprung von jdm einen Bärendienst erweisen in der Fabel „Der Bär und der Gartenliebhaber" (vgl. DUDEN 11). Diese Motiviertheit ist z.B. für die dänische Variante des Phraseologismus: gøre ngn en bjørnetjeneste durch die in den letzten Jahren stattgefundene Bedeutungsentwicklung von , ungewollt schlechter Dienst' hin zu ,guter Dienst' kaum noch vorhersagbar.

Die retrospektive Motivierbarkeit basiert auf dem Wissen der Sprecher über die Bedeutung von bekannten Phraseologismen. Durch dieses Wissen kann eine Relation zwischen der Ausdrucksseite und der Bedeutung hergestellt werden. Die folgenden Sätze beinhalten Bedeutungserklärungen ${ }^{13}$ mit expliziter retrospektiver Motivierung. Sie stammen von isländischen Sprechern, die alle angeben, den deutschen Phraseologismus selber aktiv zu verwenden: 
- Ihm ein Deutschlehrbuch schenken, hieße Eulen nach Athen tragen. Er ist doch in Deutschland aufgewachsen und spricht Deutsch wie ein Deutscher: „Überflüssiges tun, unnötiges tun, weil Ähnliches schon vorhanden. [...]. Bedeutungshinweis: Eulen, Vögel der Weisheit, hatten ihren Wohnsitz in Athen“ (Hallsteinsdóttir 2001: 243).

- Aus rein finanziellen Gründen wollen sie die Ost-Firma über die Klinge springen lassen: „Früher wurden Männer dazu verurteilt, über ein Schwert zu springen und es war Gottes oder der Götter Wille, ob sie es schafften oder nicht. Hier ist gemeint, nicht zu helfen obwohl es gut möglich wäre“(Hallsteinsdóttir 2001: 269).

- Wir müssen die Beine unter den Arm nehmen: Sich total beeilen (fast wie eine Kugel rollen, ohne Hindernis: Arme und Beine werden verschlungen miteinander) (Hallsteinsdóttir 2001: 251).

Prospektive Motivierbarkeit bezieht sich auf die Möglichkeit, in der Sprachverwendung eine Bedeutung durch die einzelnen Komponenten bzw. durch Elemente im Text zu konstruieren, d.h. es handelt sich um die Vorhersagbarkeit der Bedeutung unbekannter Phraseologismen im Text in Relation zum Sprecherwissen. So die Bedeutungserklärung eines isländischen Sprechers „Er hat was wirklich Gutes für sie getan. reiner Wein = ist auch ein guter Wein" (Hallsteinsdóttir 2001: 254) zu dem ihm unbekannten deutschen Phraseologismus im Satz: Er schenkte ihr reinen Wein ein und die Bedeutungserklärung eines englischsprachigen Sprechers "To be very open. Her heart is on her tounge: she speaks freely about things of the heart, personal, emotional things" (Hallsteinsdóttir 2001: 250) zu dem ihm ebenfalls unbekannten Phraseologismus im Satz: Sie trägt ihr Herz auf der Zunge.

\section{6. Äquivalenz}

Ein Ziel kontrastiver Arbeiten ist die Ermittlung von Äquivalenzbeziehungen (vgl. Korhonen 2007: 586). Unter Äquivalenz versteht man eine Gleichwertigkeit, die sich v.a. auf die Form, die Bedeutung und die Funktion von Sprachzeichen in zwei oder mehr Sprachen bezieht.

Allgemein wird zwischen qualitativer und quantitativer Äquivalenz unterschieden (vgl. Korhonen 2007: 577). Die quantitative Äquivalenz bezieht sich auf die Zahl der Äquivalente, wobei zwischen Monoäquivalenz, Polyäquivalenz und Nulläquivalenz unterschieden wird. Diese Art Äquivalenzbestimmung bezieht sich nur auf das phraseologische System. Andere Äquivalenzmöglichkeiten fallen prinzipiell unter die Kategorie phraseologische Nulläquivalenz 
(vgl. Abbildung 5). Die qualitative Äquivalenz (vgl. Korhonen 2007: 578579) umfasst die semantische und strukturelle Beschaffenheit der Äquivalente. Es wird zwischen Volläquivalenz (Form und Bedeutung), Teiläquivalenz (Variation der Form, Bildhaftigkeit, Metaphorik und z.T. Konnotationen und Gebräuchlichkeit) und Ersatzäquivalenz (kein systemhaftes phraseologisches Äquivalent vorhanden) unterschieden. Allein bei der Ersatzäquivalenz werden nicht-phraseologische Äquivalenzmöglichkeiten einbezogen (vgl. Korhonen 2007: 581). Eine freie syntaktische Wortverbindung, also eine Paraphrase, ist prinzipiell immer möglich. Daher sind aus einer linguistischen Perspektive v.a. äquivalente Wortbildungen und Einzellexeme interessant, hierzu gibt es allerdings kaum empirische Untersuchungen.

In vielen kontrastiven Untersuchungen sind die einzelnen phraseologischen Kategorien ein klar bevorzugter Ausgangspunkt (vgl. Malá 2005: 71). Mit einem solchen auf die Form und auf einzelne Kategorien der Phraseologie fokussierten Vergleich geht fast immer eine Kategoriengebundenheit einher in dem Sinne, dass die Einheiten einer bestimmten phraseologischen Kategorie in zwei oder mehr Sprachen verglichen werden. Fleischer (1997: 249) betont jedoch, dass eine sprachspezifische intrasprachliche Kategorie keinesfalls einen kategoriegebundenen Sprachvergleich erfordert, sondern dass sowohl in der kontrastiven Linguistik als auch intralingual die Kategorie an sich nicht für mögliche Forschungsfragen maßgebend sein sollte. Hessky (1997) meint ebenfalls, dass ein interlingualer Vergleich nur dann sinnvoll ist, wenn er von beiden Sprachen ausgeht und nicht nur als intraphraseologische „Einbahnstraße“ (Hessky 1997: 258) angesehen wird. Eine mögliche Differenzierung mit Bedeutung als Vergleichsgrundlage wird in Abbildung 6 gezeigt, zu der wir die nicht-phraseologischen Äquivalenzmöglichkeiten auch als mögliche Form in L1 hinzugefügt haben.

Die Vergleichsgrundlage der kontrastiven Phraseologie ist traditionell entweder die Bedeutung oder die Form (vgl. Korhonen 2007: 575; Reder 2006: 91), in neueren Arbeiten auch die Funktion in der Sprachverwendung (vgl. Farø 2006). So ordnen Dobrovol'skij und Piirainen (2009: 145-146) übergeordnet die relevanten zwischensprachlichen Äquivalenzaspekte ,den drei bekannten semiotischen Dimensionen - Semantik, Syntaktik (Kombinatorik) und Pragmatik“ zu. Korhonen und Wotjak (2001: 228) fordern „(e)in sorgfältig ausgearbeitetes, möglichst auch für die Computerimplementierung geeignetes, mehrdimensionales Kriterienraster als Tertium comparationis", das folgende Äquivalenzparameter beinhalten soll: „Semantik (denotativer Bedeutungskern und Konnotationen; syntagmatisch-semantische Kompatibilität und paradigmatisch-semantische Beziehungen), Lexik, Morphosyntax (praseologismusintern und -extern), Pragmatik, Bildspenderbereich, Gebrauchsüblichkeiten und 


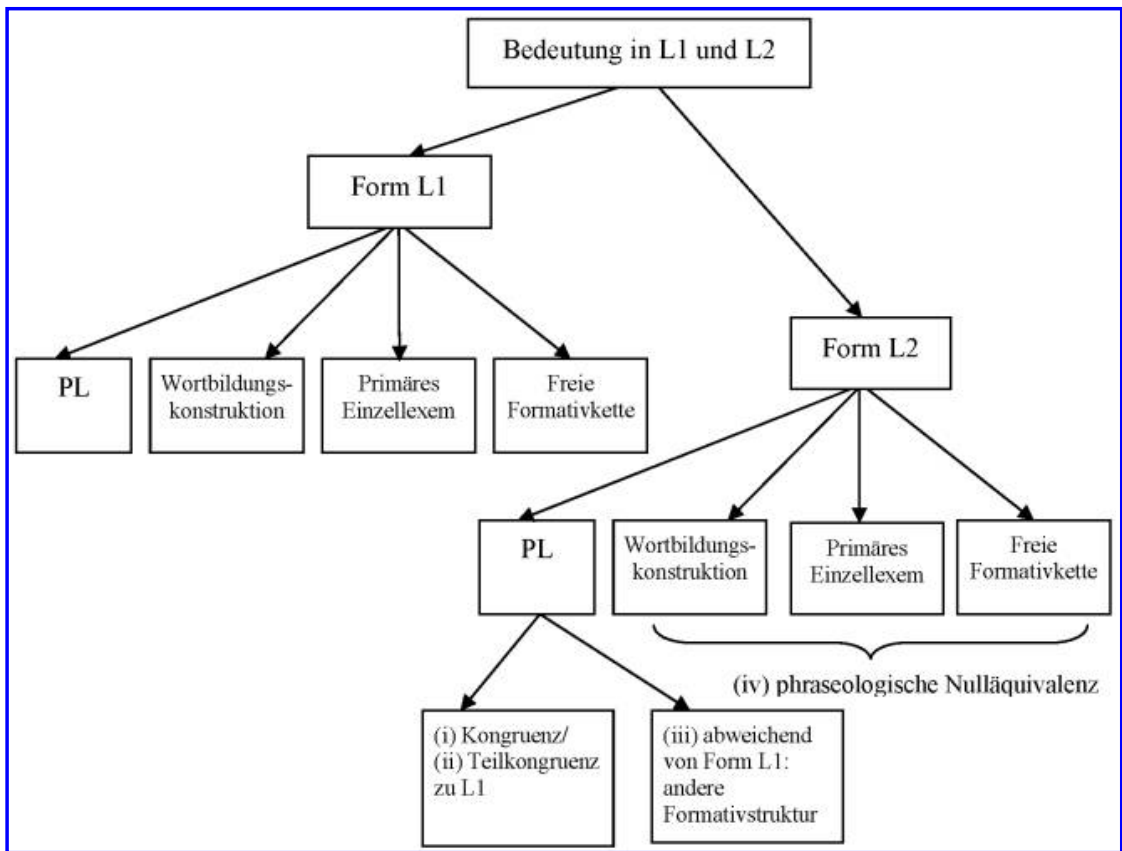

Abbildung 6. Modifizierte Darstellung der zentralen phraseologischen Äquivalenztypen nach Korhonen und Wotjak (2001: 227)

-restriktionen, Kollokabilität, Textsortenpräferenzen ... “ Und die Liste kann noch lange weitergeführt werden.

Neben der Anzahl ist die Granularität der Äquivalenzparameter in der kontrastiven Analyse ein wichtiger Aspekt, denn je feiner die Analyse ist, desto umfangreicher wird die Beschreibung. Die Relevanz einzelner Äquivalenzparameter kann erst nach einer Relevanzanalyse im konkreten Kontext festgelegt werden (vgl. Farø 2006).

Von Farø (2006) übernehmen wir einen funktionalistischen Äquivalenzbegriff, der auf der Grundlage seiner deutsch-dänischen Untersuchung entwickelt wurde und auf den folgenden Prämissen basiert:

- Die Erfassung der Äquivalenz bezieht sich immer auf das gesamte Sprachsystem, d.h. Phraseologismen, Wortbildungen und Simplizia. Eine sprachlich begründete Kategoriegebundenheit im Sinne von L1-Phraseologismus = L2Phraseologismus gibt es nicht, d.h. es gibt keine den Phraseologismen inhärente Eigenschaft, die eine Äquivalenz in Form eines anderen Phraseologismus vorschreibt (vgl. auch Fleischer 1997: 249). 
- Phraseologismen verfügen über ein besonderes semiotisches Potenzial, das sich in bestimmten Situationen entfaltet. Die Ausdrucksseite von Phraseologismen kann demzufolge eine funktionale Rolle spielen, z.B. in Verbindung mit Sprachspielen, häufig tut sie es aber in der Tat nicht. Die Konsequenz ist, dass die Ausdrucksseite für die Lexikologie, Translatologie und Lexikologie relevant sein kann, sie muss es aber nicht sein.

- Die Äquivalenz von Phraseologismen ist ein unterschiedliches Phänomen in der Lexikologie, Translatologie, Sprachdidaktik bzw. Lexikographie, das jeweils einen theoretisch-methodisch eigenen Untersuchungsgegenstand ausmacht, der aus unterschiedlichen Perspektiven betrachtet werden sollte.

In Farø (2006) werden fünf Grundbedingungen für die Bestimmung von Äquivalenz in der angewandten kontrastiven Phraseologie unterschieden:

1) Zeichentheoretische Grundbedingungen: Arbitrarität und semiotische Komplexität.

2) Disziplinspezifischer Makro- und Mikrokontext:

a. Kontrastive Lexikologie: Analyseaspekte, Form-Bedeutung, hypothetische Äquivalenzpaare.

b. Translatologie: Textfunktion, Autorenabsicht, Empfänger, Übersetzungsauftrag, textuelle Funktion des Phraseologismus.

c. Bi- und multilinguale Lexikographie: Wörterbuchkonzept, lexikographische Funktionen, Benutzervoraussetzungen, finanzielle Aspekte.

d. Fremdsprachendidaktik: Vorkenntnisse, Muttersprache, Unterrichtskonzept.

3) Granularität der Analyse: grob, mittel, fein.

4) Äquivalenzaspekte: disziplinspezifisch und von der Datengrundlage und Granularität sowie dem Ziel der Untersuchung abhängig.

5) Die lexikologische Beschreibung: die sprachsystematische Wortschatzbeschreibung ist die Voraussetzung der spezifischeren Beschreibung der angewandten Disziplinen.

Äquivalenz ist demnach ein hochkomplexer und in mehrfachem Sinn relationaler Begriff. Fragen nach Äquivalenz - sei es zu einzelnen Phraseologismen, sei es zu ganzen Inventaren - werden im Idealfall immer diese Faktoren berücksichtigen. Das heißt, dass nach der Frage nach Äquivalenz zunächst gewisse Gegenfragen beantwortet werden müssen: Innerhalb welcher linguistischen Disziplin? Wie fein granuliert soll die Beschreibung sein? Welche Äquivalenzaspekte sind relevant? Sind diese Fragen erst beantwortet und der Ausgangspunkt somit spezifiziert, dann erst lässt sie sich gezielt untersuchen. 


\section{Zu Differenzierungen des Äquivalenzbegriffs mit Blick auf die Praxis}

Eine disziplinorientierte Relevanzanalyse erfordert naturgemäß einen Zugang zur Äquivalenz via die einzelnen sprachwissenschaftlichen Disziplinen. Wir beschränken unsere folgenden Ausführungen auf die wortschatzrelevanten angewandten Disziplinen Lexikographie, Translatologie und Fremdsprachendidaktik. Die generelle Voraussetzung für Äquivalenzanalysen ist eine sprachsystematische Grundlage. Dies heißt konkret, dass die Äquivalenz zunächst als reiner lexikogrammatischer und lexiko-pragmatischer Wortschatzvergleich im Rahmen der kontrastiven Lexikologie behandelt wird. Dann erfolgt die Trennung und Spezifikation des Makro- und Mikrokontexts innerhalb des disziplinären Rahmens und nach den theoretisch-methodischen Prämissen der jeweiligen Disziplin.

Es sollte jedoch nicht erwartet werden, dass sämtliche kontrastiv-lexikologischen Daten unbedingt für alle angewandten Disziplinen relevant sind. Auf der einen Seite steht ein deskriptiv-theoretischer Vergleich und auf der anderen Seite die funktional-praktischen Arbeiten in der Lexikographie, Translatologie und Fremdsprachendidaktik mit ihren spezifischen Zielen.

\subsection{Kontrastive Lexikologie}

Die kontrastive Lexikologie umfasst Wortschatzvergleich auf der Ebene des Sprachsystems mit dem Ziel, den Wortschatz in zwei oder mehr Sprachen zu erfassen, zu beschreiben und zu vergleichen. Ein häufig verfolgtes weiteres Ziel ist es, Daten für angewandte Disziplinen zu liefern. Der Untersuchungsgegenstand sind die sprachsystematischen Eigenschaften von Phraseologismen. Die Lexikologie befasst sich - im Idealfall - mit der Erforschung und Beschreibung des gesamten Wortschatzes, von dem die Phraseologie bekanntlich einen nicht unerheblichen Teil ausmacht.

Traditionelle kontrastive lexikologische Analysen beziehen sich auf das Sprachsystem. Ihr Ausgangspunkt ist meist der Äquivalenzgrad der Bedeutung und der Form auf der Systemebene, wobei Phraseologismen ,als isolierte sprachliche Einheiten analysiert" (Koller 2007: 606) werden. Kritiker dieser Vorgehensweise haben in den letzten Jahren die Möglichkeiten computerbasierter Analyseverfahren und die Zugänglichkeit von großen Korpora genutzt, um zunächst empirisch fundierte intralinguale lexikologische Beschreibungen auszuarbeiten. Solche Untersuchungen (vgl. z.B. Fellbaum et al. 2006a, 2006b) benutzen Korpusdaten aus der Sprachverwendung, um eine sprachsystematische Beschreibung anzufertigen. Die Validierung der durch die Korpusdaten gewonnenen Informationen geschieht in einem zweiten Arbeitsschritt durch das Hinzuziehen des Sprecher(experten)wissens der einzelnen Forscher, also durch 
das induktiv-intuitive Einbeziehen der Sprachkompetenz. Diese Beschreibungen sind eine wichtige Grundlage für die kontrastive Lexikologie. Daten zur kognitiven Ebene der Sprachkompetenz der Sprecher sowie Daten zur Sprachverwendung der gesprochenen Sprache sind noch eine Seltenheit, aber im Idealfall müssten diese ergänzend zu Daten zur Sprachverwendung der geschriebenen Sprache und der Intuition der Forscher herangezogen werden.

In der kontrastiven Lexikologie werden hypothetische Äquivalenzpaare $\left(\mathrm{HÄ}{ }^{14}\right)$ auf das Vorhandensein äquivalenzrelevanter Übereinstimmungen und Abweichungen überprüft. Die lexikologische Äquivalenz kann als die vollständige Parallelität einer sehr großen Anzahl von Äquivalenzaspekten beschrieben werden, deren vollständige kontrastive Relationen im Idealfall auf der Grundlage umfassender intralingualer Korpusanalysen und Probandenbefragungen untersucht werden.

In der Phraseologie ist ein interlingualer lexikologischer Symmetriegedanke sehr verbreitet. In den Fällen, in denen bei HÄP offenbar keine Äquivalenz vorliegen konnte, hat man von „phraseologischen falschen Freunden“ gesprochen (vgl. Korhonen 2007: 584 - vgl. auch ,echte Freunde“ in Korhonen und Wotjak 2001: 227).

Wir vermuten, dass eine systematische, umfassende und empirische Untersuchung die angenommene Äquivalenz in vielen Fällen in Frage stellen würde. Dies hat zumindest die Untersuchung von Farø (2006) für äquivalente deutsch-dänische Phraseologismen festgestellt. Die große Vielfalt der Äquivalenzaspekte, deren jeweilige Relevanz sich im lexikologischen Zusammenhang prinzipiell nicht graduieren lässt, macht die lexikologische Totaläquivalenz zu einem eher unwahrscheinlichen Phänomen.

Lexikologische Äquivalenz ist keine funktionsorientierte, sondern eine systembezogene Wortschatzanalyse. ${ }^{15}$ Im Sprachsystem kann kaum abstrakt von funktionalen Äquivalenten, sondern nur von potenziell funktionalen Äquivalenzen gesprochen werden. Funktionale Äquivalente können erst in konkreten Situationen, in denen der kommunikative Zweck definiert ist, bestimmt werden.

\subsection{Translatologie}

Ein Großteil der Untersuchungen, die sich mit Phraseologie und Übersetzung beschäftigen, bezieht sich auf die Klassifikation von eingesetzten Übersetzungsäquivalenten und einer darauf aufbauenden Beschreibung von Äquivalenzproblemen. Das Hauptaugenmerk liegt auf Problemen der Übersetzung (vgl. Koller 2007), aber auch Übersetzungsmöglichkeiten werden beschrieben (vgl. Hallsteinsdóttir 1997). Für die Phraseologie werden bei Koller (2007: 610) drei Analyseperspektiven angegeben, die sich alle auf die Textebene beziehen: die 
Übersetzung als Text, der Ausgangstext und der Vergleich von Ausgangstext und Übersetzung.

Beim Übersetzen wird eine durch den Kontext monosemierte Variante der konventionell festgelegten, lexikalisierten Bedeutung eines Phraseologismus im Ausgangstext vom Übersetzer erfasst und im Zieltext wiedergegeben. Übersetzungen als Prozess sind daher laut unserer Dreiteilung an der Schnittstelle zwischen Sprachverwendung und Sprachkompetenz anzusiedeln, während Übersetzungen als Produkt zur Sprachverwendung gehören. Gemeint ist die Sprachkompetenz des jeweiligen Übersetzers sowohl in der Ausgangs- als auch in der Zielsprache, die über sein Verstehen des Ausgangstextes und die Produktion des Zieltextes entscheidet.

Translatorische Äquivalenz umfasst eine adäquate funktionale Beziehung zwischen einem Ausgangstext(teil), der einen Phraseologismus enthält, und einem Zieltext(teil). Der Zieltext vermittelt meist denselben Inhalt wie der Ausgangstext, aber die Funktionen der beiden Texte können abweichend sein. Die translatorische Äquivalenz von Phraseologismen ist als ein Teilaspekt der übergeordneten Textäquivalenz einer Übersetzung zu sehen, und sie legitimiert sich nur durch diese. Die Textäquivalenz beeinflusst somit die Äquivalenz von Phraseologismen. Die phraseologische Äquivalenz in Übersetzungen kann als eine einseitige Relation angesehen werden, die von der Phraseologie des Ausgangstexts und der Sprachkompetenz des Übersetzers gesteuert wird, ohne dass das Resultat unbedingt die gleiche Anzahl an Phraseologismen im Zieltext ist.

Ausgehend von Sprachzeichen stehen viele Möglichkeiten bei der Übersetzung zur Verfügung (vgl. Hallsteinsdóttir 1997, vgl. auch das modifizierte Modell in Abbildung 5):

- Phraseologismus - Phraseologismus

- Phraseologismus - Wort (Wortbildung oder Simplex)

- Phraseologismus - freie Wortverbindung (Paraphrase bzw. Weglassen)

- freie Wortverbindung - Phraseologismus

- Wort - Phraseologismus

Die in Farø (2006) durchgeführte Untersuchung von deutsch-dänischen literarischen Übersetzungen hat ergeben, dass sich Übersetzer bei Phraseologismen einer großen Breite an Übersetzungstypen bedienen. Tendenziell übersetzen sie jedoch kategoriegebunden Phraseologismen mit Phraseologismen. Damit beschränken sich die Übersetzer nicht selten unnötig bezüglich möglicher Lösungen und schaffen unnatürliche oder inadäquate Übersetzungen, denn es gibt keine der Phraseologie immanente Eigenschaft, die eine Übersetzung von einem Phraseologismus mit einem Phraseologismus zwingend macht. 
Die Analyse von Übersetzungen modifizierter Phraseologismen ergab eine paradoxe Konklusion: die Modifikation ist eine Realisierungsvariante von Phraseologismen, bei der ihre Form, die Ikonographie und teilweise die phraseologische Kategorie fraglos eine funktionale Rolle spielt. Gerade bei der Modifikation aber scheinen die Möglichkeiten der Übersetzung aufgrund von Ausdruck-Inhalt-Asymmetrien (vgl. Hessky 1992: 111) sehr begrenzt zu sein. Die Übersetzung modifizierter Phraseologismen ist häufig nicht auf der Wortschatz-, sondern nur auf der Text-/Inhaltsebene möglich. Modifikationen - zumindest semantische und formale, denn metakommunikative Modifikationen bereiten offenbar weniger Schwierigkeiten - können in diesem Sinn als das wirkliche Problem der Übersetzung von Phraseologismen betrachtet werden.

Eine Analyse der deutschen und der englischen Übersetzung eines Textes aus der offiziellen Touristikbroschüre der Stadt Odense von Januar $2009^{16}$ bestätigt den von Farø (2006) beschriebenen ,phraseologischen Dogmatismus“, d.h. dass die Form mit den einzelnen Komponenten und die Kategorie eindeutig einen starken Einfluss auf die Übersetzung haben:

1. På pladen har bandet rendyrket genren powerrock, som bandet gennem $3 \mathrm{EP}$ udgivelser og en masse koncerter, har gjort til deres varemærke [wörtlich: zu ihrem Warenzeichen gemacht haben, Bed. ,zu seinem Kennzeichen/ Merkmal machen '].

- Auf der Platte hat die Band in Reinkultur das Genre Powerrock entwickelt, den sie durch 3 EP-Ausgaben und viele Konzerte zu ihrem Warenzeichen gemacht hat.

- On the album, the band has cultivated the power rock genre, which has become the band's trademark, through their three EP releases and numerous concerts.

2. Begrebet dækker over en unik blanding af guitardrevet rock med kant [wörtlich: mit Kante, Bed.: 'der besonderen Art'/'mit Biss'.], melodien i centrum og et udtalt tekstunivers, hvor der ikke bliver lagt fingre imellem [wörtlich: *es werden nicht Finger zwischen gelegt, Bed.: , seine Meinung direkt sagen '].

- Der Begriff deckt eine einzigartige Mischung aus gitarrenbeheiztem Rock mit scharfen Kanten, der Melodie im Zentrum und einem ausgesprochenen Textuniversum, das keine Wünsche offen lässt.

- The concept covers a unique blend of edgy, guitar-based rock, with the melody in the centre and a pronounced lyrical universe, and the band doesn't beat about the bush!

3. Bandet har i genre tidligere både været forbi det elektroniske grænseland og følsomme ballader, men er denne gang trukket i rocktrøjen [wörtlich: 
*hat den Rockpullover angezogen, Modifikation von trcekke i arbejdstøjet, wörtlich: *die Arbeitsbekleidung anziehen, Bed.: ein Projekt, eine Aufgabe o. A. beginnen] og har skruet op for [wörtlich: *hat herauf geschraubt, Bed.: 'aufdrehen, lauter/höher stellen'] guitaren og attituden.

- Die Band hat in diesem Genre früher das elektronische Grenzland erforscht und gefühlvolle Balladen produziert. Diesmal jedoch hat sie die Rockklamotten angezogen und die Gitarre und die Attitude voll aufgedreht.

- The band has previously dabbled with a more electronic sound and sensitive ballads, but this time they have pulled on their rock caps and cranked up the guitar and attitude.

Nicht nur die einzelnen Wörter, sondern auch die Phraseologismen im Ausgangstext werden in den Zieltexten so wörtlich wie möglich übersetzt. Im Bsp. 1 werden sogar die Konstituenten der Komponente varemarke wörtlich mit Warenzeichen übersetzt, statt das lexikalisierte etw. zu seinem Markenzeichen machen zu verwenden. Diese Strategie ist ebenfalls bei der wörtlichen Übersetzung des modifizierten dänischen Phraseologismus traekke i rocktøjet und skrue op for im Bsp. 3 zu beobachten. Weiterhin wird im Bsp. 2 der ikke bliver lagt fingre imellem im Deutschen und im Englischen kategorientreu mit einem anderen Phraseologismus übersetzt. Bei rock med kant handelt es sich vermutlich um eine dänische Version des englischen with an edge, die in dänischen Wörterbüchern nicht zu finden ist, jedoch recht häufig in Verbindung mit Musik verwendet wird und im Deutschen deutlich seltener als [Musik(er)] mit scharfen Kanten vorkommt.

Zusammenfassend kann festgestellt werden: auch wenn kein Phraseologismus mit einer gleichlautenden Ausdrucksseite oder äquivalenten Bedeutung gefunden wird, wird kategorisch phraseologisch übersetzt.

Die Annahme, dass Phraseologismen normale arbiträre Sprachzeichen sind, deren Bedeutung konventionell festgelegt ist, hat für das Übersetzen einen besonderen Reiz. Damit wird erstens die häufig praktizierte Kategoriengebundenheit hinfällig und zweitens kann die Konventionalität auf weitere Ebenen der Kommunikation bezogen werden. Denn eine Übersetzung ist immer in eine zielsprachliche Kommunikation eingebunden, in der die Konventionen der Zielsprache und der Zielkultur sowie das Sprach- und Weltwissen der zielsprachigen Empfänger gelten. Welche Funktion ein Phraseologismus im Text erfüllt und somit auch seine Übersetzung, hängt in erster Linie vom Textinhalt und von den kommunikativen Konventionen des Textes ab, in dem der Phraseologismus verwendet wird - die in der Ausgangssprache und in der Zielsprache nicht unbedingt gleich sind. 


\subsection{Bilinguale Lexikographie}

Die bi- und multilinguale Lexikographie bzw. die Phraseographie (vgl. Hallsteinsdóttir 2006) beschäftigt sich mit der Darstellung der Phraseologie in zwei- oder mehrsprachigen Wörterbüchern. Als Ausgangspunkt gilt, dass zu Phraseologismen in zweisprachigen Wörterbüchern äquivalente Einheiten angegeben und sie eventuell erklärt werden.

Wir beziehen uns hier auf die Funktionslehre der Aarhuser Schule (vgl. z.B. die Übersicht in Tarp 2006 und zur Phraseographie in Hallsteinsdóttir 2009). Sie betrachtet die Frage danach, welche Benutzer welche Wörterbücher in welchen Situationen zu welchem Zweck benutzen, als die grundlegende lexikographische Frage. Das Ziel der Lexikographie ist demnach, durch die Beantwortung dieser Frage dem Wörterbuchbenutzer relevante lexikographische Informationen zu liefern.

Ein funktionaler Ausgangspunkt ist keine Selbstverständlichkeit, denn z.B. von Hanks et al. (2006: 444) wird die Beschreibung des Sprachgebrauchs als die primäre Aufgabe des Lexikographen angesehen: "The task of a lexicographer analysing a corpus is to identify all normal uses of words (not all possible uses), to group them into categories, and to offer an explanation (a "definition") of each word in each of its categories." Eine sorgfältige kontrastive lexikologische Beschreibung ist die Voraussetzung phraseographischen Arbeitens, es handelt sich dabei aber um keine lexikographische Aufgabe.

Als kommunikative Benutzungssituationen, die für zweisprachige Wörterbücher relevant sind, gelten die Sprachrezeption und -produktion in der Fremdsprache sowie das Übersetzen in die Fremdsprache oder die Muttersprache (vgl. Tarp 2006). Für die bilinguale Phraseographie bedeutet dies primär, dass Wörterbücher Informationen enthalten sollen, die dem Benutzer einerseits das Verstehen und andererseits das Verwenden von Phraseologismen ermöglichen können. Für das Verstehen reicht meist eine Bedeutungsangabe und eventuell, um das Nachvollziehen der formalen und inhaltlichen Struktur zu ermöglichen, eine komponentenbasierte - „Wort-für-Wort“-Übersetzung (vgl. Ettinger und Nunes 2006: 9). Für das Verwenden gibt Hallsteinsdóttir (2009: 221, vgl. auch Jesenšek 2006: 143, 2007) folgende lexikographische Daten an:

(a) Eine genaue Bedeutungsangabe mit einem metasprachlichen pragmatischen Kommentar zu Gebrauchsregeln und Verwendungsrestriktionen.

(b) Die Angabe der normalen syntaktischen Struktur(en).

(c) Die Angabe der Kontexte, in denen der Phraseologismus normalerweise vorkommt. 
Interlinguale lexikographische Äquivalenz betrachten wir als eine Mischung aus einer sprachsystematischen und einer funktionalen Perspektive. Funktional gesehen besteht lexikographische Äquivalenz dann, wenn mit Ausgangspunkt in einem L1-Phraseologismus ein oder mehrere L2-Sprachzeichen oder Paraphrasen dem Benutzer die für seine Benutzungssituation relevanten Informationen liefern. Phraseologische Äquivalente haben aber an sich keinen funktionalen Wert.

Dobrovol'skij und Piirainen (2009: 143) stellen fest, dass in lexikographischen Beschreibungen von einer ,prinzipiellen semantischen Äquivalenz“ ausgegangen wird. Sie konstatieren, dass sich ,in den meisten Fällen zwischensprachliche Äquivalente finden lassen, die aber andererseits so gut wie nie bedenkenlos in allen möglichen Übersetzungssituationen einsetzbar sind.“ (Dobrovol'skij und Piirainen 2009: 143). Äquivalente können bei einer Totaläquivalenz als Verstehens- und Produktionshilfe fungieren, da aber die Totaläquivalenz eher selten vorkommen dürfte, erfordert die Angabe von äquivalenten Phraseologismen sowie falschen Freunden auch eine Erklärung der Äquivalenzbeziehung.

Lexikographische Äquivalenz ist ein recht komplexer Untersuchungsgegenstand. Die Kombination der lexikologischen Beschreibung, Kotextlosigkeit, Polyfunktionalität und (printlexikographischer) Platzknappheit macht die Etablierung von wirklich anwendbaren Äquivalenzrelationen innerhalb sämtlicher (ex- oder implizit) anvisierter Wörterbuchfunktionen schwierig. Eine Bewusstwerdung über diese Komplexität ist ein notwendiger erster Schritt, der nicht zuletzt durch eigene Erfahrungen mit Vorgaben von Wörterbuchverlagen begründet ist, die durchaus noch auf eine kategoriegebundene Äquivalenz im dem Sinne bestehen, dass ein Phraseologismus mit einem Phraseologismus gleicher Kategorie erklärt werden soll. Auch wenn phraseologische Kategorien für die lexikographische Äquivalenz wenig relevant sind, können sie durchaus für den Lexikographen als ein wichtiges Hilfsmittel fungieren, weil ,unterschiedliche phraseologische Kategorien unterschiedliche Probleme bei unterschiedlichen Sprach- und Wörterbuchbenutzern verursachen können " (Farø und Lorentzen 2009: 75).

Inzwischen gibt es neben einsprachigen Projekten auch große interlinguale Wörterbuchprojekte, die die Phraseologie von einer Sprache ausgehend kontrastiv beschreiben (vgl. z.B. Durčo 2009; Jesenšek 2009). Die Darstellung der kontrastiv-zweisprachig gewonnenen Daten von äquivalenten Wortschatzeinheiten in bidirektionalen Wörterbüchern stellt nach wie vor ein Forschungsdefizit dar (vgl. Hallsteinsdóttir 2009). 


\subsection{Fremdsprachendidaktik}

Die dritte und letzte hier behandelte Schnittstelle angewandter Disziplinen zur interlingualen Phraseologie ist die Fremdsprachendidaktik, in der die Phraseologie auf allen Niveaustufen des Sprachlernens und -lehrens eine immer wichtigere Rolle spielt. ${ }^{17}$ Die Phraseodidaktik umfasst die Darstellung von Phraseologie in Lernerwörterbüchern und in Lehrwerken sowie die Entwicklung phraseodidaktischer Materialien und die Erfassung und Beschreibung der Lernervoraussetzungen. Laut Korhonen und Wotjak (2001) sollen in der Phraseodidaktik zusätzlich zu den ,phraseodidaktischen Aspekten hinsichtlich der Auswahl und Aufbereitung des Lehr- und Lernstoffs (wie Gebräuchlichkeit, Adressatenbezug und Textsortenspezifik), auch sprachenpaarbezogene Aspekte berücksichtigt werden“ (Korhonen und Wotjak 2001: 232).

Sprachdidaktische Arbeiten können übergeordnet aufgeteilt werden in:

(a) die Auswahl und Beschreibung von Phraseologie in der Sprachverwendung als Grundlage für Lehrmaterial und Lernerwörterbücher (vgl. Bergerová 2009b; Durčo 2001; Hallsteinsdóttir et al. 2006; Hessky 1992),

(b) die Methoden der Vermittlung der Phraseologie im Unterricht (vgl. va. Bergerová 2009a; Ettinger 1998, 2009; Hessky 1997; Jesenšek 2006; Kühn 1992, 1994) und die Entwicklung von Unterrichtsmaterial ${ }^{18}$ sowie

(c) die durch die Sprachkompetenz gegebenen mutter- und fremdsprachlichen Voraussetzungen der Lerner (vgl. Dobrovol'skij 1995; Hallsteinsdóttir 2001; Hessky 1987; Reder 2006, 2008).

Sprachlerner tendieren dazu, die muttersprachliche Phraseologie unreflektiert in die Fremdsprache zu übertragen. Dies geschieht sowohl in der Sprachrezeption als auch in der Sprachproduktion, weil eine durch die Ausdrucksseite der Phraseologismen bedingte Symmetrie, d.h. Äquivalenz, zwischen der Mutter- und der Fremdsprache vorausgesetzt wird. Probleme entstehen z.B. dann, wenn durch die Vorgabe der Phraseologie der Muttersprache in der Fremdsprache fehlerhafte oder nicht existente Phraseologismen produziert werden oder wenn formgleiche oder ähnliche Phraseologismen als Äquivalente interpretiert werden und deshalb die muttersprachliche Bedeutung konstruiert wird. Häufig handelt es sich um falsche Freunde, aber auch andere zwischensprachliche Unterschiede in der Bedeutung können muttersprachlich bedingte Fehler sowohl in der Sprachrezeption als auch in der Sprachproduktion verursachen. Hierzu gehören phraseologische Quasisynonyme, d.h. Phraseologismen mit gleicher oder fast gleicher bildlichen Komponente und ähnlicher Bedeutung und Phraseologismen, die zwischensprachliche asymmetrische Bedeutungsstrukturen aufweisen (vgl. Dobrovol'skij und Piirainen 2009: 146-149; Fabčič 2010). 
Eine interlinguale phraseodidaktische Äquivalenz erfordert eine Mischung aus unterschiedlichen Perspektiven. Die Grundlage für Unterrichtsmaterial ist die lexikologische Beschreibung der Phraseologie mit Fokus auf die aktuelle Sprachverwendung - also die Sprache, mit der Sprachlerner in der Tat konfrontiert werden (vgl. Hallsteinsdóttir et al. 2006). Dazu kommen Untersuchungen zur Sprachkompetenz der Lerner, um einerseits durch die Erfassung der muttersprachlichen Voraussetzungen eine gezielte Förderung und Vermeidung von Fehlern und andererseits die Einbeziehung vorhandener Verstehens- und Verarbeitungsstrategien in das Lehren und Lernen zu ermöglichen. Der starke Einfluss der muttersprachlichen Form beim Fremdsprachenlernen macht den Vergleich der Ausdrucksseite der Phraseologie (z.B. Komponentenstruktur, Ikonographie, morpho-syntaktische Eigenschaften, intrasprachlich formbedingte Wortschatzrelationen) zu einem wichtigen Forschungsgegenstand in der Phraseodidaktik.

\section{Zusammenfassung}

Unsere Unterscheidung zwischen drei Konstituierungsmöglichkeiten von Sprache: dem Sprachsystem, der Sprachverwendung und der Sprachkompetenz, ermöglicht eine differenzierte Erfassung und Beschreibung phraseologischer Eigenschaften. In Relation zu diesen Konstituierungsformen haben wir die Betrachtung von Phraseologismen in den wichtigsten interlingualen Disziplinen diskutiert, die eine Schnittstelle zur Phraseologie aufweisen. Wir haben gezeigt, dass die phraseologische Äquivalenz eine spezifisch lexikologische, translatorische, lexikographische und sprachdidaktische Perspektive hat. Diese Perspektiven sind theoretisch und methodologisch distinkt. Äquivalenz in der Phraseologie ist im Fall der Lexikologie eine sprachsystematisch-beschreibende, in der Lexikographie eine Mischung von Sprachverwendung und des Sprachsystems, in der Translatologie und der Sprachdidaktik aber in erster Linie durch die Funktion eines Phraseologismus in der Sprachverwendung und die Sprachkompetenz der einzelnen Sprecher bedingt. Zwischen der Lexikologie und den angewandten Disziplinen besteht eine einseitige Dependenzbeziehung und insofern müssen kontrastiv-lexikologische Untersuchungen als eine wichtige Grundlage der interlingualen Phraseologie betrachtet werden.

Unsere Hypothese ist, dass sich die hier beschriebenen Eigenschaften semantische Komplexität, potenzielle Mehrdeutigkeit und prinzipielle Modifizierbarkeit nicht nur in der Phraseologie im engeren Sinne, sondern auch generell in der Phraseologie beschreiben lassen. Dieser Beitrag liefert die ersten theoretisch-methodischen Überlegungen für weitere Untersuchungen dazu. 


\section{Anmerkungen}

Kontaktadressen: erla@language.sdu.dk; kenfaroe@hum.ku.dk

1. Dieser Beitrag basiert auf Ergebnissen aus Projekten, die vom DAAD, The Icelandic Research Council und Carlsbergfondet finanziert wurden. Ihnen sei hiermit für ihre großzügige Unterstützung gedankt. Für wertvolle inhaltliche Hinweise geht ein großer Dank an die beiden anonymen Gutachter.

2. Die sowjetische Forschung lieferte schon eine solide Grundlage für kontrastive Untersuchungen (vgl. Földes 2006: 12, 15). In den etwa 40 Jahren europäischer Phraseologieforschung haben viele Forscher wichtige Beiträge geliefert, z.B. Durčo (1994), Gréciano (2000), Hessky (1987), Korhonen (2007), Wotjak (1992) u.v.m. Für einen Überblick über die Forschung zu verschiedenen Sprachenpaaren vgl. Colson 2008 sowie Korhonen (2007) und Korhonen und Wotjak (2001).

3. http://www.widespread-idioms.uni-trier.de/ (30. März 2010).

4. Zur Versprachlichung von Gedanken, in sozialer Interaktion und als ästhetisches Mittel.

5. Dies wurde schon in Hallsteinsdóttir (2001: 58) problematisiert.

6. Dass dies sogar bei unbekannten Phraseologismen durchaus möglich ist, hat Hallsteinsdóttir (2001) gezeigt.

7. Im Englischen, Dänischen und Isländischen mit „(die) Zähne bürsten““.

8. www.dwds.de (21. März 2010).

9. Aus einem Bericht über ein Fußballturnier, vgl. http://www.gsvz.ch/Fussball/fussballturnier_05_lu.htm (21. März 2010).

10. www.dwds.de (21. März 2010).

11. Beispiele aus DeutscherWortschatz, www.wortschatz.uni-leipzig.de (26. März 2010)

12. Das ist natürlich unsere individuelle Interpretation; im Online-Katalog der Smithsonian Photography Initiative wird die Werbung mit den Schlagwörtern „Bulls“, „Women in advertising“, „Sex in advertising“, „Brassieres“ und „Dreams“ kategorisiert, vgl. http://photography.si.edu/SearchImage. $\operatorname{aspx} ? \mathrm{t}=3 \& \mathrm{q}=\mathrm{dreams} \& \mathrm{id}=4270 \&$ index $=2$ (30. März 2010).

13. Die Beispiele aus Hallsteinsdóttir (2001) sind authentische Bedeutungsangaben aus einer Fragebogenuntersuchung zur Bedeutung den vorangestellten kursiv gedruckten Sätzen.

14. Vgl. die HIP (Hypothetische Idiompaare) von Farø (2006).

15. Der Begriff funktionale Äquivalenz (vgl. Dobrovol'skij und Piirainen 2005) ist in Bezug auf Idiomatik in einem lexikologischen Zusammenhang nicht anwendbar, da „funktional“ in einem kontextlosen Zusammenhang ohne einen spezifischen kommunikativen Zweck nicht beschrieben werden kann.

16. Go Odense, Play Life: VisitOdenses Touristikbroschüre Januar 2009, S. 17 in der dänischen, englischen und deutschen Ausgabe.

17. Vgl. über 30 Vorträge zur Phraseodidaktik auf der IDT in Jena/Weimar im Sommer 2009: http://idt2009h6.wordpress.com/ (30. März 2010) 
18. Vgl. z.B. EPHRAS: www.ephras.org (30. März 2010) und Multimediales Unterrichtsmaterial zur deutschen Phraseologie:

http://frazeologie.ujepurkyne.com/index2.htm (30. März 2010).

\section{Literatur}

Barz, Irmhild. 2006. Wortbildung. In Die Dudenredaktion (Hg.), Duden 4. Die Grammatik, 7th edn., 641-772. Mannheim: Dudenverlag.

Barz, Irmhild. 2007. Wortbildung und Phraseologie. In Harald Burger, Dmitrij Dobrovol'skij, Peter Kühn \& Neal R. Norrick (Hg.), Phraseologie: Ein internationales Handbuch der zeitgenössischen Forschung, 27-36. Berlin \& New York: Walter de Gruyter.

Bergerová, Hana. 2009a. Wie viel Phraseologie brauchen künftige Deutschlehrende? In Libuše Spáčilová \& Lenka Vaòková (Hg.), Germanistische Linguistik - die neuen Herausforderungen in Forschung und Lehre, 71-80. Brno: Academicus.

Bergerová, Hana. 2009b. Nachdenken über ein phraseologisches Lernerwörterbuch. In Peter Ďurčo, Ružena Kozmová \& Daniela Drinková (Hg.) Deutsche Sprache in der Slowakei, 39-50. Bratislava \& Trnava: FF UCM/SUNG.

Burger, Harald. 2010. Phraseologie: Eine Einführung am Beispiel des Deutschen. Berlin: Erich Schmidt (4. Aufl.).

Burger, Harald, Annelies Buhofer \& Ambros Sialm. 1982. Handbuch der Phraseologie. Berlin \& New York: Walter de Gruyter.

Burger, Harald, Dmitrij Dobrovol'skij, Peter Kühn \& Neal R. Norrick (Hg.). 2007. Phraseologie: Ein internationales Handbuch der zeitgenössischen Forschung. Berlin \& New York: Walter de Gruyter.

Colson, Jean-Pierre. 2008. Cross-linguistic phraseological studies. An overview. In Sylviane Granger \& Fanny Meunier (Hg.), Phraseology: An interdisciplinary perspective, 191-206. Amsterdam: John Benjamins.

Colson, Jean-Pierre. 2010. The Contribution of Web-based Corpus Linguistics to a Global Theory of Phraseology. In Stefaniya Ptashnyk, Erla Hallsteinsdóttir \& Noah Bubenhofer (Hg.), Korpora, Web und Datenbanken: Computergestützte Methoden in der modernen Phraseologie und Lexikographie, 23-35. Baltmannsweiler: Schneider Verlag Hohengehren.

Dobrovol'skij, Dmitrij. 1995. Kognitive Aspekte der Idiom-Semantik: Studien zum Thesaurus deutscher Idiome. Tübingen: Narr.

Dobrovol'skij, Dmitrij. 1999. Kontrastive Phraseologie in Theorie und im Wörterbuch. In Rupprecht S. Baur, Christoph Chlosta \& Elisabeth Piirainen (Hg.), Wörter in Bildern - Bilder in Wörtern. Beiträge zur Phraseologie und Sprichwortforschung aus dem Westfälischen Arbeitskreis, 107-122. Baltmannsweiler: Schneider Verlag Hohengehren.

Dobrovol'skij, Dmitrij \& Elisabeth Piirainen. 2005. Figurative language: Cross-cultural and cross-linguistic perspectives. Amsterdam: Elsevier. 
Dobrovol'skij, Dmitrij \& Elisabeth Piirainen. 2009. Zur Theorie der Phraseologie: Kognitive und kulturelle Aspekte. Tübingen: Stauffenburg.

Donalies, Elke. 2009. Basiswissen Deutsche Phraseologie. Tübingen: Narr Francke Attempto Verlag.

Durčo, Peter. 1994. Probleme der allgemeinen und kontrastiven Phraseologie. Am Beispiel Deutsch-Slowakisch. Heidelberg: Groos.

Durčo, Peter. 2001. Bekanntheit, Häufigkeit und lexikographische Erfassung von Sprichwörtern. Zu Parömiologischen Minima für DaF. In Annelies Häcki Buhofer, Harald Burger \& Laurent Gautier (Hg.), Phraseologiae Amor: Aspekte europäischer Phraseologie, 99-106. Baltmannsweiler: Schneider Verlag Hohengehren.

Durčo, Peter. 2009. Lexikographische Beschreibung der Kollokationen kontrastiv. Vortrag auf der IDT Jena/Weimar, 3.-8. August 2009.

Ettinger, Stefan. 1998. Einige Überlegungen zur Phraseodidaktik. In Wolfgang Eismann (Hg.), EUROPHRAS 95, 201-217. Bochum: Brockmeyer.

Ettinger, Stefan \& Manuela Nunes. 2006. Portugiesische Redewendungen. Hamburg: Buske Helmut Verlag.

Fabčič, Melanija Larisa. 2009. Eine kognitiv-semantische Interpretation der phraseologischen Äquivalenz am Beispiel der EPHRAS-Datenbank (Kontrast: DeutschSlowenisch). In Csaba Földes (Hg.), Phraseologie disziplinär und interdisziplinär, 423-432. Tübingen: Gunter Narr Verlag.

Farø, Ken. 2004. Hvornår går man over åen efter vand? Idiomatiske ækvivalensproblemer i leksikologi og leksikografi [Wann geht man über den Fluss um Wasser $\mathrm{zu}$ holen? Idiomatische Äquivalenzprobleme in Lexikologie und Lexikographie]. LexicoNordica $11,85-108$

Farø, Ken. 2006. Idiomatizität - Ikonizität - Arbitrarität: Beitrag zu einer funktionalistischen Theorie der Idiomäquivalenz. Kopenhagen: Doktorarbeit an der Universität Kopenhagen.

Farø, Ken \& Erla Hallsteinsdóttir. 2006. Neue theoretische und methodische Ansätze in der Phraseologieforschung: Einleitung zum Themenheft, Linguistik online 27, 2/06, 3-10. www.linguistik-online.de/27_06/einleitung.pdf(30. März 2010).

Farø, Ken \& Henrik Lorentzen. 2009. De oversete og mishandlede ordforbindelser hvilke, hvor og hvorfor? [Übersehene und mißhandelte Wortverbindungen - welche, wo und warum?], LexicoNordica 16, 75-101.

Fellbaum, Christiane, Undine Kramer \& Gerald Neumann. 2006. Korpusbasierte lexikografische Erfassung und linguistische Analyse deutscher Idiome. In Annelies Häcki Buhofer \& Harald Burger (Hg.), Phraseology in Motion I: Methoden und Kritik, 43-56. Baltmannsweiler: Schneider Verlag Hohengehren.

Fellbaum, Christiane, Fabian Koerner \& Gerald Neumann. 2006. Corpus-based studies of German idioms and light verbs. International Journal of Lexicography 19(4). 349-360.

Fleischer, Wolfgang. 1997. Phraseologie der deutschen Gegenwartssprache. Tübingen: Niemeyer (2. Aufl.).

Földes, Csaba. 1997. Konzepte der kontrastiven Phraseologie. Kontrastiv, Germanistisches Jahrbuch für Nordeuropa 15. 167-182. 
Földes, Csaba. 2006. Deutsche Phraseologie kontrastiv: Intra- und interlinguale Zugänge. Heidelberg: Julius Groos Verlag.

Földes, Csaba. 2007. Phraseologismen und Sprichwörter im Kontext von Mehrsprachigkeit und Transkulturalität: eine empirische Studie. Proverbium 24. 119-152.

-Fuhrhop, Nanna. 2008. Das graphematische Wort (im Deutschen): Eine erste Annäherung. Zeitschrift für Sprachwissenschaft 27, 189-228.

Gréciano, Gertrud. 2000. Phraseologie: Spezifische Merkmale, intra- und interlingual. Revista de Filología Alemana 8. 233-251.

Gries, Stefan Th. 2008. Phraseology and linguistic theory: A brief survey. In Sylviane Granger \& Fanny Meunier (Hg.), Phraseology: An interdisciplinary perspective, 3-26. Amsterdam: John Benjamins.

Häcki Buhofer, Annelies. 2006. Vorwort. In Annelies Häcki Buhofer \& Harald Burger (Hg.), Phraseology in Motion I: Methoden und Kritik, I-XVI. Baltmannsweiler: Schneider Verlag Hohengehren.

Hallsteinsdóttir, Erla. 1997. Aspekte der Übersetzung von Phraseologismen am Beispiel Isländisch - Deutsch. In Eberhard Fleischmann, Wladimir Kutz \& Peter A. Schmitt (Hg.), Translationsdidaktik: Grundfragen der Übersetzungswissenschaft, 561-569. Tübingen: Gunter Narr.

Hallsteinsdóttir, Erla. 2001. Das Verstehen idiomatischer Phraseologismen in der Fremdsprache Deutsch. Hamburg: Verlag Dr. Kovač.

www.verlagdrkovac.de/0435_volltext.htm (30. März 2010).

Hallsteinsdóttir, Erla. 2006. Phraseographie. Hermes: Journal of Language and Communication Studies 36. 91-128.

Hallsteinsdóttir, Erla. 2007. Wörtliche, freie und phraseologische Bedeutung. Eine korpusbasierte Untersuchung des Vorkommens von freien und phraseologischen Lesarten bei deutschen Idiomen. In Erika Kržišnik \& Wolfgang Eismann (Hg.), Phraseologie in der Sprachwissenschaft und anderen Disziplinen, 151-165. Ljubljana: Univerze v Ljubljani.

Hallsteinsdóttir, Erla. 2009. Zweisprachige Lernerlexikografie aus funktionaler Sicht. In Carmen Mellado Blanco (Hg.), Theorie und Praxis der idiomatischen Wörterbücher, 209-232. Tübingen: Max Niemeyer Verlag.

Hallsteinsdóttir, Erla. 2009. Wörtliche, freie und phraseologische Bedeutung. Eine korpusbasierte Untersuchung von freien und phraseologischen Lesarten bei deutschen idiomen. In Csaba Földes (Hg.), Phraseologie disziplinär und interdisziplinär, 145-154. Tübingen: Gunter Narr Verlag.

Hallsteinsdóttir, Erla, Monika Sajankova \& Uwe Quasthoff. 2006. Vorschlag eines phraseologischen Optimums für Deutsch als Fremdsprache auf der Basis von Frequenzuntersuchungen und Geläufigkeitsbestimmungen. Linguistik-online 27, 2/06, 119-138. http://www.linguistik-online.de/27_06/hallsteinsdottir_et_al.pdf (30. März 2010).

Hanks, Patrick, Anne Urbschat \& Elke Gehweiler. 2006. German light verb constructions in corpora and dictionaries. International Journal of Lexicography 19 (4). $439-457$. 
Hessky, Regina. 1987. Phraseologie: Linguistische Grundfragen und kontrastives Modell deutsch-ungarisch. Tübingen: Max Niemeyer.

Hessky, Regina. 1992. Phraseolexeme als harte Nuß für die zweisprachige Lexikographie. In Csaba Földes (Hg.), Deutsche Phraseologie in Sprachsystem und Sprachverwendung, 107-124. Wien: Edition Praesens.

Hessky, Regina. 1997. Einige Fragen der Vermittlung von Phraseologie im Unterricht Deutsch als Fremdsprache. In Rainer Wimmer \& Franz-Josef Berens (Hg.), Wortbildung und Phraseologie, 245-261. Tübingen: Gunter Narr.

Hessky, Regina \& Stefan Ettinger. 2009. Deutsche Redewendungen: Ein Wörter- und Übungsbuch für Fortgeschrittene. Stark erweiterte Internetfassung als ,,phraseologisches Lesebuch“. http://www.ettinger-phraseologie.de/(30. März 2010).

Jesenšek, Vida. 2006. Phraseologie und Fremdsprachenlernen: Zur Problematik einer angemessenen phraseodidaktischen Umsetzung. Linguistik online 27, 2/06. 137-147.

Jesenšek, Vida. 2007. Zum Problem der Äquivalenz in mehrsprachigen phraseologischen Wörterbüchern. In Annelies Häcki Buhofer \& Harald Burger (Hg.), Phraseology in Motion II: Theorie und Anwendung, 275-286. Baltmannsweiler: Schneider Verlag Hohengehren.

Jesenšek, Vida. 2009. Phraseologische Wörterbücher auf dem Weg zu Phraseologiedatenbanken. In Carmen Mellado Blanco (Hg.), Theorie und Praxis der idiomatischen Wörterbücher, 65-82. Tübingen: Max Niemeyer Verlag.

Juska-Bacher, Britta. 2009. Empirisch-kontrastive Phraseologie: Am Beispiel der Bekanntheit der Niederländischen Sprichwörter im Niederländischen, Deutschen und Schwedischen. Baltmannsweilser: Schneider Verlag Hohengehren.

Kilian, Jörg. 2001. Kritische Semantik: Für eine wissenschaftliche Sprachkritik im Spannungsfeld von Sprachtheorie, Sprachnorm, Sprachpraxis. Zeitschrift für germanistische Linguistik 29. 293-318.

Koller, Werner. 2007. Probleme der Übersetzung von Phrasemen. In Harald Burger, Dmitrij Dobrovol'skij, Peter Kühn \& Neal R. Norrick (Hg.), Phraseologie: Ein internationales Handbuch der zeitgenössischen Forschung, 605-613. Berlin, New York: Walter de Gruyter.

Korhonen, Jarmo. 2007. Probleme der kontrastiven Phraseologie. In Harald Burger, Dmitrij Dobrovol'skij, Peter Kühn \& Neal R. Norrick (Hg.), Phraseologie: Ein internationales Handbuch der zeitgenössischen Forschung, 574-598. Berlin, New York: Walter de Gruyter.

Korhonen, Jarmo \& Barbara Wotjak. 2001. Kontrastivität in der Phraseologie. In Gerhard Helbig, Lutz Gotze \& Gert Henrici (Hg.), Deutsch als Fremdsprache: ein internationales Handbuch, 224-235. Berlin: de Gruyter.

Kühn, Peter. 1992. Phraseodidaktik: Entwicklungen, Probleme und Überlegungen für den Muttersprachenunterricht und den Unterricht Deutsch als Fremdsprache. Fremdsprachen lehren und lernen 21. 169-186.

Kühn, Peter. 1994. Pragmatische Phraseologie: Konsequenzen für die Phraseographie und Phraseodidaktik. In Barbara Sandig (Hg.), EUROPHRAS 92: Tendenzen der Phraseologieforschung, 411-428. Bochum: Brockmeyer. 
Malá, Jiøina. 2005. Phraseologie: Blütezeit einer linguistischen Disziplin (Eine Reise durch die Phraseologielandschaft). Brünner Beiträge zur Germanistik und Nordistik 10. $65-77$.

Mellado Blanco, Carmen. 2009. Intensivierung durch Vergleich im Deutschen und Spanischen: Intralinguale und kontrastive Analyse der semantischen Beziehungen unter den Vergleichskomponenten. In Csaba Földes (Hg.), Phraseologie disziplinär und interdisziplinär, 465-476. Tübingen: Gunter Narr Verlag.

Mieder, Wolfgang. 2009. International bibliography of paremiology and phraseology. Berlin \& New York: de Gruyter.

Piirainen, Elisabeth. 1999. Falsche Freunde in der Phraseologie des Sprachenpaares Deutsch - Niederländisch. In Annette Sabban (Hg.), Phraseologie und Übersetzen: Phrasemata II, 187-204. Bielefeld: Aisthesis.

Piirainen, Elisabeth. 2008. Phraseology in a European framework: A cross-linguistic and cross-cultural research project on widespread idioms. In Sylviane Granger und Fanny Meunier (Hg.), Phraseology: An interdisciplinary perspective, 243-258. Amsterdam: John Benjamins.

Reder, Anna. 2006. Kollokationen in der Wortschatzarbeit. Wien: Praesens Verlag.

Reder, Anna. 2008. Erkennen DaF-Lernende Kollokationen? In György Scheibl (Hg.), Tests im DaF-Unterricht-DaF-Unterricht im Test, 105-115. Szeged: Grimm.

Sabban, Annette. 2007. Culture-boundness and problems of cross-cultural phraseology. In Harald Burger, Dmitrij Dobrovol'skij, Peter Kühn \& Neal R. Norrick (Hg.), Phraseologie: Ein internationales Handbuch der zeitgenössischen Forschung, 590-605. Berlin, New York: Walter de Gruyter.

Sinclair, John. 1991. Corpus, concordance, collocation. Oxford: Oxford University Press.

Sinclair, John. 2008. The phrase, the whole phrase, and nothing but the phrase. In Sylviane Granger \& Fanny Meunier(Hg.), Phraseology: An interdisciplinary perspective, 407 410. Amsterdam: John Benjamins.

Soehn, Jan-Philipp (2006): Über Bärendienste und erstaunte Bauklötze: Idiome ohne freie Lesart in der HPSG. Frankfurt a/M: Peter Lang.

Soehn, Jan-Philipp \& Christine Römer. 2007. Wann ist ein Idiom ein Idiom? Eine Analyse von Phraseologismen ohne freie Lesart. In Annelies Häcki Buhofer \& Harald Burger (Hg.), Phraseology in Motion II: Theorie und Anwendung. Akten der Internationalen Tagung zur Phraseologie (Basel, 2004), 3-14. Baltmannsweiler: Schneider Verlag Hohengehren,

Tarp, Sven. 2006. Leksikografien i grcenselandet mellem viden og ikke-viden: Generel leksikografisk teori med sarlig henblik på lørnerleksikografi [Die Lexikographie im Grenzland zwischen Wissen und Nicht-Wissen: Eine generelle Theorie der Lexikographie mit Fokus auf Lernerlexikographie]. Aarhus: Handelshøjskolen i Aarhus.

Wotjak, Barbara. 1992. Verbale Phraseolexeme in System und Text. Tübingen: Max Niemeyer. 